\title{
Existence and approximation of solutions to an anisotropic phase field system for the kinetics of phase transitions
}

\author{
OLAF KLEIN ${ }^{\dagger}$ \\ Weierstrass Institute for Applied Analysis and Stochastics, Mohrenstrasse 39, D-10117 Berlin, \\ Germany
}

[Received 25 July 2000 and in revised form 13 July 2001]

\begin{abstract}
This paper is concerned with a phase field system of Penrose-Fife type for a non-conserved order parameter with a kinetic relaxation coefficient depending on the gradient of the order parameter. This system can be used to model the anisotropic solidification of liquids. A time-discrete scheme for an initial-boundary value problem to this system is presented. By proving the convergence of this scheme, the existence of a solution to the problem is shown.
\end{abstract}

Keywords: Phase-field model; anisotropy; semidiscretization; convergence

\section{Introduction}

A class of phase-field systems modelling the dynamics of diffusive phase transitions has been derived by Penrose and Fife in [32]. Dealing with a non-conserved order parameter, one of these systems is generalized to the following form:

$$
\begin{aligned}
c_{0} \theta_{t}+\lambda^{\prime}(\chi) \chi_{t}+\nabla \vec{q} & =g, \quad \vec{q}=\kappa(\theta) \nabla\left(\frac{1}{\theta}\right), \\
\zeta(\nabla \chi) \chi_{t}-\varepsilon \Delta \chi+s^{\prime}(\chi) & =-\frac{\lambda^{\prime}(\chi)}{\theta} .
\end{aligned}
$$

In this system an energy balance (1.1) is coupled with an evolution (1.2) for the order parameter $\chi$. These equations determine the evolution of the absolute temperature $\theta$ and the order parameter. Here, the positive constant $c_{0}$ is the specific heat, and the function $\kappa$, which attains only positive values, models some temperature-dependent thermal conductivity. The heat flux $\vec{q}$ satisfies the Fourier law, if $\kappa(\theta)=\kappa_{\text {Four }} \theta^{2}$ with some positive constant $\kappa_{\text {Four }}$. The function $\lambda^{\prime}(\chi)$ represents the latent heat of the phase transition, and the datum $g$ represents heat sources or sinks.

Moreover, $\zeta$ stands for a kinetic relaxation coefficient depending on the gradient of the order parameter, the positive constant $\varepsilon$ is a relaxation coefficient, representing the energy of the phase interfaces, and $s^{\prime}$ is the derivative of some potential on $\mathbb{R}$.

In the context of a solid-liquid phase transition with a critical temperature $\theta_{C}$, one typically has a quadratic or linear function $\lambda$ and the potential $s(r)$ is the sum of $\frac{\lambda(r)}{\theta_{C}}$ and the double-well potential $\varrho\left(r^{2}-1\right)^{2}$ with some positive constant $\varrho$. To ensure that the order parameter attains only values in the interval $[-1,1]$, the double-obstacle potential $I_{[-1,1]}(r)+\varrho\left(1-r^{2}\right)$, with $I_{[-1,1]}$ being the indicator function of the interval $[-1,1]$, is used instead of the double-well potential.

Email: klein@wias-berlin.de 
In this work, the existence of a solution to an initial-boundary value problem for the PenroseFife (PF) system (1.1), (1.2) is proved by considering a time-discrete scheme and proving the convergence of the scheme. These results can be applied for the situations discussed above.

The existence of solutions to the PF system with a constant kinetic relaxation parameter $\zeta$ has been investigated in a number of papers, for example in $[20-22,26,28,29,35,38]$ for $\kappa$ being a constant, and, for more general heat flux laws, in $[9,10,12,30]$. In $[14,23,24]$, such investigations have been performed for the PF system with $\zeta$ and $\kappa$ being constant and $\theta_{t}$ being replaced by the time derivative of a more general function of $1 / \theta$. In $[25,27]$ the numerical approximation of $\mathrm{PF}$ systems with $\kappa$ being a constant and a space depending kinetic relaxation parameter $\zeta$ has been considered.

To the knowledge of the author, a dependence of this kinetic relaxation parameter on the gradient of the order parameter has not been taken into account before in the context of a PF system. For the standard phase field system (see [6]), i.e. the system (1.1), (1.2) with $\lambda^{\prime}(\chi) \equiv L$ for some constant $L>0$, a Fourier law heat flux, i.e. $\vec{q}=-\kappa_{\text {Four }} \nabla \theta$, and $\frac{1}{\theta}$ in the order parameter equation (1.2) replaced by $-\theta$, this has already been done: see, for example, $[7,16]$.

A kinetic relaxation parameter $\zeta$ depending on the gradient of the order parameter allows one to model the evolution of a phase interface with a direction-dependent kinetic mobility, i.e. a phase interface whose normal velocity depends on orientation of the phase interface. If such a kinetic mobility is used, and the growth of a solid phase into a surrounding liquid phase is considered, an anisotropic growth process will be observed, i.e. the solid phase will grow faster in some directions, and slower in other directions. On first glance, one would assume that this would lead to the growth of tips in the directions with a higher growth speed, and therefore to the formation of dendrites, but in the performed numerical simulations this has not been observed. Instead, these simulations have produced a behaviour that is similar to the one described in [33: Section 2.3]: during the growing of the solid phase, the part of the phase interphase with an orientation corresponding to a higher growth speed grows out and becomes smaller, while the part with a lower growth speed orientation becomes bigger. After some time, the phase interphase consists only of regions with orientations in the slow growing regime, separated by corners.

If only (1.2) with a given right-hand side and $s$ equal to the double-well or the double-obstacle potential is considered, one is dealing with the Allen-Cahn equation or the double-obstacle AllenCahn equation, respectively. In these models, one does not take into account the latent heat of the phase transitions. The double-obstacle Allen-Cahn equation with a kinetic relaxation parameter $\zeta$ depending on the direction of $\nabla \chi$ is considered in [17-19].

To deal with non-smooth potentials, the potential is split in the form $s(r)=\phi(r)-\sigma(r)$, where $\phi$ represents the convex, maybe not smooth, part of the potential, and $\sigma$ is a differentiable function, such that $-\sigma$ can represent the non-convex part of the potential. Now, in (1.2), $s^{\prime}(\chi)$ is replaced by $\xi-\sigma^{\prime}(\chi)$, where $\xi$ is a representation of the subdifferential $\partial \phi$ of $\phi$.

Moreover, the heat flux will be written in the form $\vec{q}=-\nabla \alpha(\theta)$. For a function $\alpha$ such that $\alpha^{\prime}(\theta) \theta^{2}=\kappa(\theta)$, we get the heat flux considered in (1.1). The Fourier law is obtained by using $\alpha(\theta)=\kappa_{\text {Four }} \theta$.

The layout of this paper is as follows. In Section 2, the formulation of the considered initialboundary value problem for phase-field system and two existence results are presented. In Section 3, the time-discrete scheme is introduced and the approximation results are shown. Sections 4-7 deal with the proof of the results. Their contents are discussed at the end of Section 3. 


\section{The phase-field system}

In this section, an initial-boundary value problem for the phase field system of PF type is investigated. It will be considered on a bounded, open domain $\Omega \subset \mathbb{R}^{N}$ with $N \in\{2,3\}$ and a smooth boundary $\Gamma=\partial \Omega$. Let $\Omega_{T}:=\Omega \times(0, T)$ and $\Gamma_{T}:=\Gamma \times(0, T)$, where $T>0$ stands for a final time.

First, the boundary condition for the temperature is derived. In Section 2.2, a way to deal with $1 / \theta$ and $\alpha(\theta)$ is introduced, which is also valid if $\theta$ is not a function on $\Omega$ but only a functional on some function space on $\Omega$. This way is used in the formulation of the initial-boundary value problem in Section 2.3. An existence result is presented in Section 2.4, and a stronger existence result, which requires additional assumptions, is presented in Section 2.5.

\subsection{Boundary condition for the temperature}

On the boundary $\Gamma$, a heat exchange with an external environment at temperature $\theta_{\text {ext }}$ is considered. For an energy balance with a heat flux $\vec{q}$ satisfying the Fourier law with a constant thermal conductivity $\kappa_{\text {Four }}>0$, i.e. $\vec{q}=-\kappa_{\text {Four }} \nabla \theta$, this is modelled by the boundary condition

$$
-\kappa_{\text {Four }} \frac{\partial \theta}{\partial n}=\gamma_{\text {Four }}\left(\theta-\theta_{\text {ext }}\right) \text {, }
$$

where $\gamma_{\text {Four }}$ is some positive constant and $n$ is the outward unit normal to $\Gamma$.

Now, the derivation of this boundary condition as the author learned from [37] is adapted to deal with more general heat fluxes. By considering the following computations for the special form $\alpha(\theta)=\kappa_{\text {Four }} \theta$, which correspond to the Fourier law, one gets the boundary condition (2.1).

To avoid technicalities, the derivation is presented in a one-dimensional, time-independent setup. Hence, we consider a temperature field $\theta$ on an interval $\left(x_{0}, x_{1}\right)$.

The external temperature $\theta_{\text {ext }}\left(x_{1}\right)$ considered on the right-hand side $x_{1}$ of the interval does not correspond to a physical temperature of the environment in the point $x_{1}$, but to the temperature on the right-hand side of some interfacial region on the right-hand side of $x_{1}$, wherein the physical temperature changes continuously its value from $\theta\left(x_{1}\right)$ to $\theta_{\text {ext }}\left(x_{1}\right)$.

We assume that the interfacial region has the thickness $\delta>0$. Hence, we can extend the temperature field $\theta$ continuously to $\left[x_{0}, x_{1}+\delta\right]$ such that

$$
\theta\left(x_{1}+\delta\right)=\theta_{\mathrm{ext}}\left(x_{1}\right)
$$

Since the heat flux is continuous across $\partial\left(x_{0}, x_{1}\right)$, we have $q\left(x_{1}\right)=q_{\text {inter }}\left(x_{1}\right)$, where $q$ denotes the the heat flux in $\left[x_{0}, x_{1}\right]$ and $q_{\text {inter }}$ denotes the heat flux in the interfacial region, i.e. in $\Omega_{\text {inter }}:=$ $\left[x_{1}, x_{1}+\delta\right]$. Assuming that the heat flux stays constant inside the interfacial region, we get

$$
q\left(x_{1}\right)=q_{\text {inter }}\left(x_{1}+\tau\right), \quad \forall 0 \leqslant \tau \leqslant \delta .
$$

To derive the boundary condition for (1.1) with an heat flux of the form $\vec{q}=-\nabla \alpha(\theta)$, we consider in $\left(x_{0}, x_{1}\right)$ the corresponding heat flux $q=-\frac{\partial}{\partial x}(\alpha(\theta))$ and in $\Omega_{\text {inter }}$ a heat flux of the same form for a function $\alpha_{\text {inter }}=\gamma_{\text {inter }} \alpha$ with some positive constant $\gamma_{\text {inter }}$. Hence, we have $q_{\text {inter }}=$ $-\gamma_{\text {inter }} \frac{\partial}{\partial x}(\alpha(\theta))$ and (2.3) yields that

$$
-\frac{\partial \alpha(\theta)}{\partial x}\left(x_{1}\right)=q\left(x_{1}\right)=-\gamma_{\text {inter }} \frac{\partial \alpha(\theta)}{\partial x}\left(x_{1}+\tau\right), \quad \forall 0 \leqslant \tau \leqslant \delta .
$$


Hence, considering the integral over $\left[x_{1}, x_{1}+\delta\right]$ and using (2.2), we observe that

$$
\delta \frac{\partial \alpha(\theta)}{\partial x}\left(x_{1}\right)=\gamma_{\text {inter }}\left(\alpha\left(\theta_{\text {ext }}\left(x_{1}\right)\right)-\alpha\left(\theta\left(x_{1}\right)\right)\right) .
$$

Defining $\gamma:=\frac{\gamma_{\text {inter }}}{\delta}$ and performing an analogous calculation also for the left-hand side of the interval, we get on $\partial\left(x_{0}, x_{1}\right)$ :

$$
\frac{\partial \alpha(\theta)}{\partial n}=\gamma\left(\alpha\left(\theta_{\mathrm{ext}}\right)-\alpha(\theta)\right) .
$$

Hence, we have derived a boundary condition that models the heat exchange through a thin interface surrounding the considered domain, with a heat flux inside this interface that is proportional to the considered heat flux $\vec{q}=\nabla \alpha(\theta)$ in the domain. To the knowledge of the author, the physical meaning of this boundary condition has not been pointed out until now, even if this boundary condition has already been used in a number of papers dealing with PF systems: see, for example, [11,25-27] for the special form $\alpha(\theta)=-\kappa_{0} / \theta$ with some positive constant $\kappa_{0}$, and $[9,10,14,23,24]$ for more general $\alpha$.

Performing the same computations as above for a heat flux with thermal memory, which means to replace $\alpha(\theta)$ by a function depending also on the former values of the temperature $\theta$, one gets the boundary condition for the heat flux which is used in [12].

Considering (2.4) on $\Gamma_{T}$ and defining $\mu: \Gamma_{T} \rightarrow \mathbb{R}$ by $\mu:=\gamma \alpha\left(\theta_{\mathrm{ext}}\right)$, we get the boundary condition that is used in this work.

\subsection{New formulation to deal with $1 / \theta$ and $\alpha(\theta)$}

Following an approach used in a number of papers dealing with PF systems, the considered system is rewritten by introducing two additional unknowns $u$ and $w$. The $1 / \theta$-term and the $\alpha(\theta)$-term in the system are replaced by $u$ and $w$ respectively, and the additional compatibility conditions $u=1 / \theta$ and $w=\alpha(\theta)$ a.e. on $\Omega_{T}$ are used. To prepare the weak formulation of the PF system, these conditions have to be replaced by weaker ones, which can also be applied if $\theta(t)$ is a not function on $\Omega$ but only a functional in $H^{1}(\Omega)^{*}$. The following assumptions for $\alpha$ are intended to be used in the existence results, and one could replace these assumptions by weaker ones, if one is only interested in the reformulation of the compatibility conditions.

(A1) Let $\alpha \in C^{1}((0, \infty))$ be an increasing function, with $\alpha^{\prime}$ non-increasing, such that there exist positive constants $\theta_{\text {low }}, \alpha_{\text {low }}, \alpha_{\text {factor }}$, and $\alpha_{\text {factor }}^{\prime}$ with

$$
\alpha(s)=-\frac{\alpha_{\text {low }}}{s}, \quad \forall s \in\left(0, \theta_{\text {low }}\right], \quad \alpha(r) \geqslant-\alpha_{\text {factor }} \frac{1}{r}, \quad \alpha^{\prime}(r) \geqslant \alpha_{\text {factor }}^{\prime} \frac{1}{r^{2}} \quad \forall r>0 .
$$

$$
\text { Let } \tilde{\alpha}(s):=\int_{\theta_{\text {low }}}^{s} \alpha(r) \mathrm{d} r \text {. }
$$

REMARK 2.1 The heat flux laws corresponding to assumption (A1) can represent at high temperatures a Fourier law, while there has to be a singularity at $\theta=0$. This singularity is used to get $a$ priori estimates for $1 / \theta$ such that one can control the singularity at $\theta=0$ appearing in the right-hand side of the order parameter equation. These heat flux laws are a special case of the heat flux laws considered in $[9,10]$, where instead of (2.5) more general conditions have been considered. 
The PF system is intended to be used at intermediate temperatures. Therefore, the thermodynamic functions and material laws used in the derivation of this system are only valid in a certain temperature range. For example, a PF system which models a solid-liquid phase transition is not supposed to be valid for temperature above the boiling temperature where the liquid is transformed to vapour. For dealing with the evaporation one has to use another model. Therefore, one can choose $\theta_{\text {low }}^{*}, \theta_{\text {high }}^{*} \in(0, \infty)$, such that the interval $\left[\theta_{\text {low }}^{*}, \theta_{\text {high }}^{*}\right]$ encloses the temperature range in which the PF system is valid.

For a function $\alpha_{*}$ such that the conditions in (A1), apart from (2.5), are satisfied, one can define a function $\alpha$ satisfying (A1) such that $\nabla \alpha_{*}(\theta)$ and $\nabla \alpha(\theta)$ lead to the same heat flux, as long as the temperature stays inside $\left[\theta_{\text {low }}^{*}, \theta_{\text {high }}^{*}\right.$. Hence, the heat flux $\nabla \alpha(\theta)$ can be considered as regularization of $\nabla \alpha_{*}(\theta)$, where the regularization only takes place for temperatures outside the temperature range where the PF system is supposed to be valid.

For $\tilde{w}$ and $\tilde{\theta}$ in $L^{2}(\Omega)$ the conditions $\tilde{\theta}>0$ and $\tilde{w}=\alpha(\tilde{\theta})$ a.e. on $\Omega$ are equivalent to $\tilde{w} \in \partial j_{0}(\tilde{\theta})$ in $L^{2}(\Omega)$, where $j_{0}: L^{2}(\Omega) \mapsto \mathbb{R} \cup\{+\infty\}$ is the $L^{2}(\Omega)$-representation of the convex function $\tilde{\alpha}:(0, \infty) \rightarrow \mathbb{R}$. We have for $f \in L^{2}(\Omega)$

$$
j_{0}(f):= \begin{cases}\int_{\Omega} \tilde{\alpha}(f(x)) \mathrm{d} x, & \text { if } f>0 \text { a.e. in } \Omega \text { and } \tilde{\alpha}(f) \in L^{1}(\Omega) \\ +\infty, & \text { otherwise. }\end{cases}
$$

In $[14,15,23]$, Damlamian, Kenmochi, and Kubo extend this function to a function on $H^{1}(\Omega)^{*}$ and replaced the $L^{2}(\Omega)$-compatibility condition by a condition in this space. Following their formulation, we denote by $V$ the Hilbert space, arising by considering $H^{1}(\Omega)$ with the inner product $(\cdot, \cdot)_{V}$ defined by

$$
(w, v)_{V}=\int_{\Omega} \nabla w \bullet \nabla v \mathrm{~d} x+\gamma \int_{\Gamma} w v \mathrm{~d} \sigma, \quad \forall w, v \in H^{1}(\Omega),
$$

and the corresponding norm $\|\cdot\|_{V}$. Thanks to the trace theorem and Poincaré's inequality, we see that the norms $\|\cdot\|_{V}$ and $\|\cdot\|_{H^{1}(\Omega)}$ are equivalent. Hence, $V^{*}$ can be identified with $H^{1}(\Omega)^{*}$ and the $H^{1}(\Omega)^{*}$-norm is equivalent to the induced norm $\|\cdot\|_{V^{*}}$ on $V^{*}$ as dual space of $V$. Let $F: V \rightarrow V^{*}$ be the duality mapping:

$$
\langle F w, v\rangle_{V^{*} \times V}=(w, v)_{V}, \quad \forall w, v \in V
$$

We see that $V^{*}$ is a Hilbert space with the inner product $(\cdot, \cdot)_{*}$

$$
(w, v)_{*}:=\left\langle w, F^{-1} v\right\rangle_{V^{*} \times V}=\left(F^{-1} w, F^{-1} v\right)_{V}, \quad \forall w, v \in V^{*},
$$

satisfying

$$
\|f\|_{V^{*}}=\sqrt{(f, f)_{*}}=\left\|F^{-1} f\right\|_{V}, \quad \forall f \in V^{*} .
$$

Now, $j_{0}$ is extended to work on the whole $V^{*}$ by considering the corresponding $\Gamma$-regularization $j$ of $j_{0}$ on $V^{*}$, i.e. we have

$$
j(w)=\inf \left\{\liminf _{n \rightarrow \infty} j_{0}\left(z_{n}\right):\left(z_{n}\right)_{n \in \mathbb{N}} \subset L^{2}(\Omega), \quad z_{n} \rightarrow z \text { in } V^{*}\right\}, \quad \forall w \in V^{*} .
$$


Replacing in these definitions $\alpha$ by the function $(0, \infty) \ni s \mapsto-1 / s$, which also satisfies (A1), we get two other convex, lower semicontinuous functionals $\tilde{J}_{0}: L^{2}(\Omega) \rightarrow \mathbb{R} \cup\{\infty\}$ and $\tilde{J}$ : $V^{*} \rightarrow \mathbb{R} \cup\{\infty\}$. We have, see [15: Theorem 1.5, Corollary 1.6], with $\partial_{*} j$ and $\partial_{*} \tilde{J}$ denoting the subdifferential of $j$ and $J$ respectively in the Hilbert space $V^{*}$ :

LEMMA 2.2 Assume that (A1) holds. We have $j=j_{0}$ and $\tilde{J}=\tilde{J}_{0}$ on $L^{2}(\Omega)$.

For $\tilde{\theta} \in L^{2}(\Omega)$

$$
\begin{gathered}
\tilde{\theta} \in D\left(\partial_{*} j\right) \Leftrightarrow \tilde{\theta}>0 \text { a.e. in } \Omega \quad \text { and } \quad \exists \tilde{w} \in H^{1}(\Omega): \tilde{w}=\alpha(\tilde{\theta}), \quad \text { a.e. in } \quad \Omega, \\
\tilde{\theta} \in D\left(\partial_{*} \tilde{J}\right) \Leftrightarrow \tilde{\theta}>0 \text { a.e. in } \Omega \quad \text { and } \quad \exists \tilde{u} \in H^{1}(\Omega):-\tilde{u}=-1 / \tilde{\theta}, \quad \text { a.e. in } \quad \Omega .
\end{gathered}
$$

We have $\partial_{*} j(\tilde{\theta})=\{F \tilde{w}\}$ with $\tilde{w}$ as in (2.12) for $\tilde{\theta} \in L^{2}(\Omega) \cap D\left(\partial_{*} j\right)$. We have $\partial_{*} \tilde{J}(\tilde{\theta})=\{-F \tilde{u}\}$ with $\tilde{u}$ as in (2.13) for $\tilde{\theta} \in L^{2}(\Omega) \cap D\left(\partial_{*} J\right)$.

\subsection{The phase-field system}

We consider the following initial-boundary value problem for the phase field system of PF type.

(PF) Find $(\theta, w, u, \chi, \xi)$ fulfilling

$$
\begin{gathered}
\theta \in H^{1}\left(0, T ; V^{*}\right), \quad w, u \in L^{2}(0, T ; V), \\
\chi \in H^{1}\left(0, T ; L^{2}(\Omega)\right) \cap L^{2}\left(0, T ; H^{2}(\Omega)\right), \quad \xi \in L^{2}\left(0, T ; L^{2}(\Omega)\right), \\
F w(t) \in \partial_{*} j(\theta(t)), \quad-F u(t) \in \partial_{*} J(\theta(t)), \quad \text { in } V^{*}, \quad \text { for a.e. } t \in(0, T), \\
\chi \in D(\beta), \quad \xi \in \beta(\chi), \quad \text { a.e. in } \Omega_{T} . \\
\left\langle c_{0} \theta_{t}(t), v\right\rangle_{V^{*} \times V}+\int_{\Omega} \lambda^{\prime}(\chi(t)) \chi_{t}(t) v \mathrm{~d} x+\int_{\Omega} \nabla w(t) \bullet \nabla v \mathrm{~d} x+\gamma \int_{\Gamma} w(t) v \mathrm{~d} \sigma \\
=\int_{\Omega} g(t) v \mathrm{~d} x+\int_{\Gamma} \mu(t) v \mathrm{~d} \sigma, \quad \forall v \in V, \quad \text { for a.e. } t \in(0, T), \\
\zeta(\nabla \chi) \chi_{t}-\varepsilon \Delta \chi+\xi-\sigma^{\prime}(\chi)=-\lambda^{\prime}(\chi) u, \quad \text { a.e. in } \quad \Omega_{T}, \\
\quad \frac{\partial \chi}{\partial n}=0, \quad \text { a.e. in } \Gamma_{T}, \\
\quad \text { in } V^{*}, \quad \chi(\cdot, 0)=\chi^{0}, \quad \text { a.e. in } \Omega .
\end{gathered}
$$

REMARK 2.3 We see that (2.14e) is the weak formulation of the first equation in (1.1) for $\vec{q}=$ $-\nabla \alpha(\theta)$, combined with the boundary condition (2.4) for $\mu:=\gamma \alpha\left(\theta_{\mathrm{ext}}\right)$.

REMARK 2.4 If (A1) holds, and a solution to the problem (PF) with $\theta \in L^{2}\left(0, T ; L^{2}(\Omega)\right.$ ) is given, we get from Lemma 2.2 that the compatibility conditions between $\theta, u$, and $w$, holds a.e. on $\Omega_{T}$, i.e.

$$
\theta>0, \quad w=\alpha(\theta), \quad u=\frac{1}{\theta}, \quad \text { a.e. in } \quad \Omega_{T} .
$$

\subsection{First existence result}

For dealing with the system (PF), in addition to (A1), the following assumptions will be used. 
(A2) Let $\beta$ be a maximal monotone graph on $\mathbb{R}$ and $\phi: \mathbb{R} \rightarrow[0, \infty]$ a convex, lower semicontinuous function satisfying

$$
\beta=\partial \phi, \quad 0 \in D(\beta), \quad 0 \in \beta(0), \quad \text { int } D(\beta) \neq \emptyset .
$$

(A3) There are positive constants $\lambda_{\text {factor }}, \sigma_{\text {factor }}^{\prime}, \lambda_{\text {sup }}^{\prime \prime}, \sigma_{\text {sup }}^{\prime \prime}$ such that

$$
\begin{aligned}
\lambda & \in W_{\text {loc }}^{2, \infty}(\mathbb{R}), \quad \sigma \in W_{\text {loc }}^{2, \infty}(\mathbb{R}), \\
-\lambda(s) & \leqslant \lambda_{\text {factor }}(\phi(s)+1), \quad\left(\sigma^{\prime}(s)\right)^{2} \leqslant \sigma_{\text {factor }}^{\prime}(\phi(s)+1), \quad \forall s \in D(\beta), \\
\left|\lambda^{\prime \prime}(s)\right| & \leqslant \lambda_{\text {sup }}^{\prime \prime}, \quad\left|\sigma^{\prime \prime}(s)\right| \leqslant \sigma_{\text {sup }}^{\prime \prime}, \quad \text { for a.e. } s \in \mathbb{R} .
\end{aligned}
$$

(A4) We have

$$
g \in L^{2}\left(0, T ; L^{2}(\Omega)\right), \quad \mu \in L^{2}\left(0, T ; H^{\frac{1}{2}}(\Gamma)\right) .
$$

(A5) We consider initial data $\theta^{0} \in L^{2}(\Omega), \chi^{0} \in H^{2}(\Omega)$, such that

$$
\theta^{0}>0, \quad \chi^{0} \in D(\beta), \quad \text { a.e. in } \quad \Omega, \quad \phi\left(\chi^{0}\right), \tilde{\alpha}\left(\theta^{0}\right), \ln \left(\theta^{0}\right) \in L^{1}(\Omega) .
$$

(A6) We have positive constants $\zeta_{\text {inf }}, \zeta_{\text {sup }}$ such that the function $\zeta: \mathbb{R}^{N} \rightarrow\left[\zeta_{\text {inf }}, \zeta_{\text {sup }}\right]$ is continuous on $\mathbb{R}^{N}$.

We have the following existence result, which is the main result of this work.

THEOREM 2.5 Assume that (A1)-(A6) hold. Then there is a solution $(\theta, w, u, \chi, \xi)$ to the PF system.

REMARK 2.6 Similar to the existence result for the phase relaxation system with a kinetic relaxation parameter depending on the gradient of the order parameter studied in [8], no uniqueness result is known for the solution to $(\mathrm{PF})$.

REMARK 2.7 If one starts to model the evolution of a phase interface with a kinetic mobility term depending on orientation of the interface, one would like to use a kinetic relaxation parameter $\zeta$ which depends only on the direction of $\nabla \chi$, and has therefore a discontinuity in 0 , but neither one of the theorems in this section nor the later Theorem 3.4 apply to this situation.

By extending the concept of $L^{p}(\Omega)$-viscosity solution as in [5] with considerations similar to Chapter 9 in [13], we get a $L^{p}(\Omega)$-viscosity solution formulation for (2.14f), which is also valid if $u$ and $\zeta$ are not continuous. But, also in this formulation, $\chi$ has to be continuous on $\Omega_{T}$, and the continuity of $\chi$ could not even be shown for the solution to the PF system with continuous $\zeta$ (see Theorems 2.5 and 2.8).

\subsection{Second existence result}

In addition to the result above, one can also show that there is a solution to the PF system, such that $\theta$ is function on $\Omega_{T}$, at least under the following additional assumptions: 
(A7) We have a positive constant $\alpha_{\text {low }}$ such that

$$
\alpha(r)=-\alpha_{\text {low }} \frac{1}{r}, \quad \forall r>0 .
$$

(A8) We have a positive constant $\lambda_{\text {sup }}^{\prime}$ such that

$$
\left|\lambda^{\prime}(s)\right| \leqslant \lambda_{\text {sup }}^{\prime}, \quad \forall s \in D(\beta), \quad \mu \leqslant 0, \quad \text { a.e. in } \quad \Gamma_{T} .
$$

The existence results reads as follows.

TheOREM 2.8 Assume that (A2)-(A8) hold. Then there is a solution $(\theta, w, u, \chi, \xi)$ to the PF system with $\theta \in L^{\infty}\left(0, T ; L^{2}(\Omega)\right)$ and (2.15).

REMARK 2.9 Assumption (A7) corresponds to the heat flux $\vec{q}=-\alpha_{\text {low }} \nabla(1 / \theta)$, which is also investigated in [20-22, 26, 28, 29, 35, 38]. This heat flux is not considered because of some special physical significance, but because of its properties which simplify the mathematical investigations. These properties are used in the proof of Theorem 2.8. One can also derive an existence result with $\theta \in L^{\infty}\left(0, T ; L^{2}(\Omega)\right)$ under somehow weaker assumptions for the heat flux, but this would require more technicalities.

REMARK 2.10 If (A8) is satisfied, the latent heat function $\lambda^{\prime}(\chi)$ is bounded. Hence, for a quadratic $\lambda$, the assumption (A8) can only be satisfied if $D(\beta)$ is bounded. This is the case for the doubleobstacle potential, but not for the double-well potential. For dealing with this situation, one has to consider the existence result presented earlier in Theorem 2.5.

For $\alpha$ as in (A7), a function $\theta_{\text {ext }}$ on $\Gamma_{T}$ with $\theta_{\text {ext }}>0$, and $\mu$ defined as at the end of Section 2.1, i.e. $\mu:=\gamma \alpha\left(\theta_{\text {ext }}\right)$, we have $\mu<0$, i.e. the assumption for $\mu$ in (A8) is satisfied.

\section{The time-discrete scheme}

In this section, a time-discrete scheme is introduced to prepare numerical computations. Moreover, this scheme is used to prove the existence results in the last section. We consider time-step sizes that do not need to be uniform, but satisfy the following assumption, where $c_{\text {up }} \geqslant 1$ is a fixed constant.

(A9) The vector $H=\left(h_{1}, \ldots, h_{K}\right) \in \mathbb{R}^{K}$ of time-step sizes, with $K \in \mathbb{N}$, fulfils

$$
\begin{gathered}
\sum_{m=1}^{K} h_{m}=T, \quad h_{m} \leqslant c_{\text {up }} h_{m-1}, \quad \forall 1<m \leqslant K, \\
0<h_{m}<\frac{\zeta_{\text {inf }}}{3 \sigma_{\text {sup }}^{\prime \prime}}, \quad \forall 1 \leqslant m \leqslant K .
\end{gathered}
$$

We define $h_{\max }(H):=\max _{1 \leqslant m \leqslant K} h_{m}, t_{0}:=0$, and, for $1 \leqslant m \leqslant K$ :

$$
\begin{gathered}
t_{m}:=t_{m-1}+h_{m}=\sum_{i=1}^{m} h_{i}, \\
g_{m}(x):=\frac{1}{h_{m}} \int_{t_{m-1}}^{t_{m}} g(x, t) \mathrm{d} t, \quad \mu_{m}(\sigma):=\frac{1}{h_{m}} \int_{t_{m-1}}^{t_{m}} \mu(\sigma, t) \mathrm{d} t, \quad \forall x \in \Omega, \sigma \in \Gamma .
\end{gathered}
$$

Now, an Euler scheme in time for the PF systems is presented, which is implicit, except for the treatment of the nonlinearities $\lambda^{\prime}, \sigma^{\prime}$, and $\zeta$. 
(D) Let

$$
\theta_{0}:=\theta^{0}, \quad \chi_{0}:=\chi^{0},
$$

and, for $1 \leqslant m \leqslant K$, find

$$
\theta_{m} \in L^{2}(\Omega), \quad w_{m} \in H^{2}(\Omega), \quad u_{m} \in H^{1}(\Omega), \quad \chi_{m} \in H^{2}(\Omega), \quad \xi_{m} \in L^{2}(\Omega),
$$

such that

$$
\begin{gathered}
0<\theta_{m}, \quad \alpha\left(\theta_{m}\right)=w_{m}, \quad u_{m}=\frac{1}{\theta_{m}}, \quad \chi_{m} \in D(\beta), \quad \xi_{m} \in \beta\left(\chi_{m}\right), \quad \text { a.e. in } \Omega, \\
c_{0} \frac{\theta_{m}-\theta_{m-1}}{h_{m}}+\lambda^{\prime}\left(\chi_{m-1}\right) \frac{\chi_{m}-\chi_{m-1}}{h_{m}}-\Delta w_{m}=g_{m}, \quad \text { a.e. in } \quad \Omega, \\
\zeta\left(\nabla \chi_{m-1}\right) \frac{\chi_{m}-\chi_{m-1}}{h_{m}}-\varepsilon \Delta \chi_{m}+\xi_{m}-\sigma^{\prime \prime}\left(\chi_{m-1}\right) \chi_{m} \\
=-\lambda^{\prime}\left(\chi_{m-1}\right) u_{m}-\sigma^{\prime \prime}\left(\chi_{m-1}\right) \chi_{m-1}+\sigma^{\prime}\left(\chi_{m-1}\right), \quad \text { a.e. in } \quad \Omega, \\
\frac{\partial w_{m}}{\partial n}=\mu_{m}-\gamma w_{m}, \quad \frac{\partial \chi_{m}}{\partial n}=0, \quad \text { a.e. in } \quad \Gamma .
\end{gathered}
$$

REMARK 3.1 The time-discrete scheme (D), especially the approximation used for the coupling terms, is chosen in such a way that one can use discrete versions of the a priori estimates derived by Sprekels and Zheng [35].

The approximation for $\sigma^{\prime}\left(\chi_{m}\right)$ used in (3.4d) is linear with respect to $\chi_{m}$, i.e. to the implicit part, and involves an approximation error which is less or equal to $\sigma_{\text {sup }}^{\prime \prime}\left(\chi_{m}-\chi_{m-1}\right)^{2}$. This approximation is equal to $\sigma^{\prime}\left(\chi_{m}\right)$, if $\sigma^{\prime}(\cdot)$ is an affine function.

TheOREM 3.2 Assume that (A1)-(A6), and (A9) hold.

(1) Then there exists a solution to (D).

(2) The solution is unique, if (A7) holds.

REMARK 3.3 For heat flux laws corresponding to (A7) the right-hand side of the later contracting inequality (6.2) is zero, while for a more general heat flux, i.e. a more general $\alpha$, one has to estimate this right-hand side to get a uniqueness result. Therefore, to avoid technicalities, the uniqueness result in the last theorem is only proved under the assumption (A7).

We use a solution to (D) to construct an approximate solution $(\widehat{\theta}, \bar{w}, \bar{u}, \widehat{\chi}, \bar{\xi})$ in $\left(L^{\infty}\left(0, T ; L^{2}(\Omega)\right)\right)^{4}$ to the PF system. The function $\widehat{\theta}$ is defined to be linear in time on $\left[t_{m-1}, t_{m}\right]$ for $m=1, \ldots, K$ such that $\widehat{\theta}\left(t_{k}\right)=\theta_{k}$ holds for $k=0, \ldots, K$. The function $\widehat{\chi}$ is defined analogously. We define $\bar{w}$ piecewise constant in time by $\bar{w}(t)=w_{m}$ for $t \in\left(t_{m-1}, t_{m}\right]$ and $m=1, \ldots, K$. The functions $\bar{u}, \bar{\xi}$ are defined analogously.

We have the following convergence result.

THEOREM 3.4 Assume that (A1)-(A6) hold. Let a sequence $\left\{H^{(n)}\right\}_{n \in \mathbb{N}}$ of vectors of time-step sizes with (A9) and $h_{\max }\left(H^{(n)}\right) \underset{n \rightarrow \infty}{\longrightarrow} 0$ be given. Denote by $\left(\left(\widehat{\theta}^{(n)}, \bar{w}^{(n)}, \bar{u}^{(n)}, \widehat{\chi}^{(n)}, \bar{\xi}^{(n)}\right)\right)_{n \in \mathbb{N}}$ a corresponding sequence of approximations. Hence, there is a subsequence $\left\{n_{k}\right\}_{k \in \mathbb{N}}$ and a solution 
$(\theta, w, u, \chi, \xi)$ to the PF system such that

$$
\begin{array}{ll}
\widehat{\theta}^{\left(n_{k}\right)} \underset{k \rightarrow \infty}{\longrightarrow} \theta, & \text { strongly in } \quad C\left([0, T] ; V^{*}\right), \\
& \text { weakly in } H^{1}\left(0, T ; V^{*}\right), \\
\bar{w}^{\left(n_{k}\right)} \underset{k \rightarrow \infty}{\longrightarrow} w, & \text { weakly in } L^{2}(0, T ; V), \\
\bar{u}^{\left(n_{k}\right)} \underset{k \rightarrow \infty}{\longrightarrow} u, & \text { weakly in } L^{2}(0, T ; V), \\
\widehat{\chi}^{\left(n_{k}\right)} \underset{k \rightarrow \infty}{\longrightarrow} \chi, & \text { weakly in } H^{1}\left(0, T ; L^{2}(\Omega)\right) \cap L^{2}\left(0, T ; H^{2}(\Omega)\right), \\
\bar{\xi}^{\left(n_{k}\right)} \underset{k \rightarrow \infty}{\longrightarrow} \xi, & \text { weakly-star in } L^{\infty}\left(0, T ; H^{1}(\Omega)\right),
\end{array}
$$

If also (A7) and (A8) are satisfied, then we have

$$
\widehat{\theta}^{\left(n_{k}\right)} \underset{k \rightarrow \infty}{\longrightarrow} \theta, \quad \text { weakly-star in } \quad L^{\infty}\left(0, T ; L^{2}(\Omega)\right) .
$$

REMARK 3.5 The upper bound for $\lambda^{\prime \prime}$ and $\sigma^{\prime \prime}$ used in (A3) can be weakened to some growth condition by using the same approach as in [27].

A crucial step in the proof is the derivation of the strong $L^{6}\left(0, T ; H^{1}(\Omega)\right)$ convergence (see (7.10)) for the approximation of $\chi$. To avoid therein a more technical argumentation as in the derivation of [8: (3.29)], it is assumed in (A5) that $\chi^{0} \in H^{2}(\Omega)$. One can weaken this regularity assumption to $\chi^{0} \in H^{1}(\Omega)$ and derive the same results, except for (3.9): instead of the weak $L^{2}\left(0, T ; H^{2}(\Omega)\right)$ convergence for $\widehat{\chi}^{\left(n_{k}\right)}$ only a weak convergence in $L^{2}\left(t, T ; H^{2}(\Omega)\right)$ for all $0<t<T$ could be shown.

REMARK 3.6 If (A3) holds, there is some $n_{0} \in \mathbb{N}$ satisfying $3 T \sigma_{\text {sup }}^{\prime \prime} \leqslant n_{0} \zeta_{\text {inf }}$. For $n \in \mathbb{N}$, we can consider the vector $H^{(n)}=\left(h^{(n)}, h^{(n)}, \ldots, h^{(n)}\right) \in \mathbb{R}^{n+n_{0}}$ of time-step sizes with $h^{(n)}:=$ $\frac{T}{n_{0}+n}$, such that (A9) is satisfied. Hence, it follows from Theorem 3.4 that Theorem 2.5 is satisfied. Since (A1) follows from (A7), we get Theorem 2.8 by combining Theorem 3.4 with Remark 2.4.

In the sequel, first Theorem 3.2 will be proved, following the proof of [27: Theorem 2.1]. Under the additional assumption that $D(\beta)$ is bounded, the existence of a solution to the scheme is proved in Section 4, and uniform estimates for the solutions to the scheme are derived in Section 5. In Section 6 , these results are combined to derive the existence and uniqueness results for the timediscrete scheme.

In Section 7, the convergence of the solutions to the time-discrete scheme and the existence of a solution to the considered PF system is proved. So, Theorem 3.4 is proved. In the sequel, the notation $\|\cdot\|_{p}$ will be used for the $L^{p}(\Omega)$-norm and the notation $\|\cdot\|_{p, N}$ will be used for the $\left(L^{p}(\Omega)\right)^{N}$-norm for all $p \in[1, \infty]$.

REMARK 3.7 As mentioned in Remark 2.7, one is interested in weakening the assumption (A6) on the kinetic relaxation parameter $\zeta$ by allowing $\zeta$ to be discontinuous in 0 . If this weaker version of (A6) is used, Theorem 3.2 still holds and the estimates in Section 5 can be performed for the corresponding solutions to the scheme. Hence, one can get all convergence results in Section 6 except that of (7.14), and one is therefore not able to prove in this way that (2.14f) is satisfied. 


\section{The existence of a solution to the scheme (D) for $D(\beta)$ bounded}

Before presenting the existence result, some properties for $\alpha$ and $\alpha^{-1}$ are derived. Here, $R(\alpha)=$ $\{\alpha(s): s>0\}$ denotes the range of the function $\alpha$.

Lemma 4.1 Assume that (A1) holds. There is a positive constant $C_{\alpha}^{\prime}$, such that

$$
|\tilde{\alpha}(s)| \leqslant \tilde{\alpha}(s)+C_{\alpha}^{\prime}(1+|\ln (s)|), \quad \forall s>0 .
$$

Moreover, the inverse function $\psi:=\alpha^{-1}: R(\alpha) \rightarrow(0, \infty)$ is an increasing $C^{1}$-function, such that $\psi^{\prime}$ is non-decreasing. The function $\varrho: \mathbb{R} \rightarrow(0, \infty)$ with

$$
\varrho(w):= \begin{cases}1 / \psi(w), & \text { if } w \in R(\alpha), \\ 0, & \text { otherwise, }\end{cases}
$$

is Lipschitz-continuous and decreasing on $\mathbb{R}$, and $C^{1}$ on $R(\alpha)$. There is a positive constant $C_{\varrho}$, such that

$$
\begin{gathered}
\psi(w) w \geqslant-\alpha_{\text {factor }}, \quad \varrho^{\prime}(w)=-\frac{1}{(\psi(w))^{2} \alpha^{\prime}(\psi(w))}, \quad \forall w \in R(\alpha), \\
0>\varrho^{\prime}(w) \geqslant-\frac{1}{\alpha_{\text {factor }}^{\prime}}, \quad 0 \leqslant \varrho(w) \leqslant \frac{1}{\theta_{\text {low }}}+\frac{1}{\alpha_{\text {low }}}|w|, \quad \forall w \in R(\alpha), \\
\alpha_{\text {factor }}^{\prime}\left(\varrho^{\prime}(w)\right)^{2} \leqslant-\varrho^{\prime}(w), \quad \alpha_{\text {low }}(\varrho(w))^{2} \leqslant C_{\varrho}-w \varrho(w), \quad \forall w \in R(\alpha) .
\end{gathered}
$$

Proof. Thanks to (A1), we see that $\tilde{\alpha} \geqslant 0$ on $\left(0, \theta_{\text {low }}\right)$, and that for $s \geqslant \theta_{\text {low }}$ it holds that

$$
-\tilde{\alpha}(s) \leqslant \int_{\theta_{\text {low }}}^{s} \alpha_{\text {factor }} \frac{1}{r} \mathrm{~d} r=\alpha_{\text {factor }}\left(\ln s-\ln \theta_{\text {low }}\right) .
$$

This leads to (4.1). Invoking (A1), we deduce that $r \alpha(r) \geqslant-\alpha_{\text {factor }}$ for all $r>0$. Hence, we see that the inequality in (4.3) holds, while the equality follows from the definitions of $\varrho$ and $\psi$ by applying the chain rule. Combining this equality with (A1) and $\left(\varrho^{\prime}(w)\right)^{2}=\left(-\varrho^{\prime}(w)\right)\left(-\varrho^{\prime}(w)\right)$, we see that the estimates for $\varrho^{\prime}$ in (4.4) and (4.5) hold. Because of (A1), we have

$$
\begin{gathered}
\varrho(w)=-\frac{w}{\alpha_{\text {low }}}, \quad(\varrho(w))^{2}=-\varrho(w) \frac{w}{\alpha_{\text {low }}}, \quad \forall w \leqslant-\frac{\alpha_{\text {low }}}{\theta_{\text {low }}} . \\
\varrho(w) \leqslant \varrho\left(\frac{-\alpha_{\text {low }}}{\theta_{\text {low }}}\right)=\frac{1}{\theta_{\text {low }}}, \quad-w \varrho(w)=-\frac{\alpha(\psi(w))}{\psi(w)}, \quad \forall w \geqslant-\frac{\alpha_{\text {low }}}{\theta_{\text {low }}} .
\end{gathered}
$$

Recalling (A1) leads to

$$
\alpha(s) \leqslant \alpha\left(\theta_{\text {low }}\right)+\alpha^{\prime}\left(\theta_{\text {low }}\right)\left(s-\theta_{\text {low }}\right) \leqslant \frac{\alpha_{\text {low }}}{\theta_{\text {low }}^{2}}, \quad \forall s \geqslant \theta_{\text {low }} .
$$

Combining this with (4.6) and (4.7) leads to the estimates for $\varrho$ in (4.4) and (4.5).

We have the following existence result.

Lemma 4.2 Assume that (A2)-(A6), (A9) hold, and that $D(\beta)$ is bounded. Then, there exists a solution to the scheme (D). 
Proof. The existence of a solution to the scheme will be shown by induction. Thanks to (3.3), (A5), and Sobolev's embedding theorem, we have $\theta_{0} \in L^{2}(\Omega), \chi_{0} \in L^{\infty}(\Omega)$. Let $\theta_{m-1} \in L^{2}(\Omega)$ and $\chi_{m-1} \in L^{\infty}(\Omega)$ be given for some $m \in\{1, \ldots, K\}$. Because of (A3) and (A6), we obtain $\lambda^{\prime}\left(\chi_{m-1}\right) \in L^{\infty}(\Omega)$ and $\zeta\left(\nabla \chi_{m-1}\right) \in L^{\infty}(\Omega)$. To rewrite the conditions in the scheme, let the nonlinear operators $A_{m}$ and $B_{m}$ on $L^{2}(\Omega)$ and the linear operator $D_{m}: L^{2}(\Omega) \rightarrow L^{2}(\Omega)$ be defined by

$$
\begin{aligned}
A_{m} w= & c_{0} \psi(w)-h_{m} \Delta w-c_{0} \theta_{m-1}-h_{m} g_{m} \\
& -\lambda^{\prime}\left(\chi_{m-1}\right) \chi_{m-1}, \quad \text { a.e. in } \Omega, \quad \forall w \in D\left(A_{m}\right), \\
D\left(A_{m}\right)= & \left\{w \in H^{2}(\Omega) \mid \frac{\partial w}{\partial n}=\mu_{m}-\gamma w, \quad \text { a.e. in } \quad \Gamma, \quad \psi(w) \in L^{2}(\Omega)\right\}, \\
B_{m} \chi= & -\varepsilon \Delta \chi+\left\{\xi \in L^{2}(\Omega) \mid \xi \in \beta(\chi), \quad \text { a.e. in } \quad \Omega\right\}, \\
D\left(B_{m}\right)= & \left\{\chi \in H^{2}(\Omega) \mid \frac{\partial \chi}{\partial n}=0, \quad \text { a.e. in } \quad \Gamma, \quad \chi \in D(\beta), \quad \text { a.e. in } \quad \Omega,\right. \\
& \left.\exists \xi \in L^{2}(\Omega): \xi \in \beta(\chi), \quad \text { a.e. in } \quad \Omega\right\}, \\
D_{m} \chi= & \left(\frac{\zeta\left(\nabla \chi_{m-1}\right)}{h_{m}}-\frac{\zeta_{\text {inf }}}{2 h_{m}}-\sigma^{\prime \prime}\left(\chi_{m-1}\right)\right) \chi, \quad \text { a.e. in } \quad \Omega, \quad \forall \chi \in L^{2}(\Omega) .
\end{aligned}
$$

Thanks to (A6), (A3), (A9), and $\chi_{m-1} \in L^{2}(\Omega)$, we conclude that $D_{m}$ is a maximal monotone linear operator, and [4: Corollary 13] yields that $B_{m}$ is a maximal monotone operator on $L^{2}(\Omega)$.

By translating the proof of [4: Corollary 13], we see that the operator $A_{m}$ is maximal monotone. Using (4.3), we can show that this operator is also coercive. Hence, we obtain (see, for example, [36: Chapter I, Theorem 2.4]) that the operator is also surjective. By finally estimating the difference between two given solutions, we have shown that $A_{m}$ is one-to-one as operator from $D\left(A_{m}\right) \rightarrow$ $L^{2}(\Omega)$. The proof is similar for the one for $\alpha(\theta)=-\kappa_{0} / \theta$ in [25: Lemma 5.1].

Using $\lambda^{\prime}\left(\chi_{m-1}\right) \in L^{\infty}(\Omega)$ and that $A_{m}$ is one-to-one as operator from $D\left(A_{m}\right) \rightarrow L^{2}(\Omega)$, we deduce that for every $\chi \in L^{2}(\Omega)$ there is a unique function $w \in D\left(A_{m}\right)$ such that

$$
A_{m} w=-\lambda^{\prime}\left(\chi_{m-1}\right) \chi
$$

is satisfied. Defining now $W(\chi):=w$, we get an operator $W$ from $L^{2}(\Omega) \rightarrow H^{2}(\Omega)$.

Let $f_{m} \in L^{2}(\Omega)$ be defined by

$$
f_{m}:=\frac{1}{h_{m}} \zeta\left(\nabla \chi_{m-1}\right) \chi_{m-1}-\sigma^{\prime \prime}\left(\chi_{m-1}\right) \chi_{m-1}+\sigma^{\prime}\left(\chi_{m-1}\right), \quad \text { a.e. in } \quad \Omega .
$$

Since $D\left(D_{m}\right)=L^{2}(\Omega)$, we have $D\left(B_{m}\right) \cap$ int $D\left(D_{m}\right) \neq \emptyset$. Hence, applying a theorem on summing maximal monotone operators (see, for example, [3: Chapter II, Theorem 1.7]), we see that $B_{m}+D_{m}$ is maximal monotone. For $w \in L^{2}(\Omega)$ and $\varrho$ as in (4.2), we get from (A3), $\chi_{m-1} \in H^{2}(\Omega)$, and (4.4) that $\lambda^{\prime}\left(\chi_{m-1}\right) \varrho(w) \in L^{2}(\Omega)$. Therefore, there is a unique function $\chi \in D\left(B_{m}+D_{m}\right)$ such that

$$
\frac{\zeta_{\text {inf }}}{2 h_{m}} \chi+B_{m} \chi+D_{m} \chi+\lambda^{\prime}\left(\chi_{m-1}\right) \varrho(w) \ni f_{m}
$$


Setting $\Psi(w):=\chi$, we get an operator from $L^{2}(\Omega)$ to $D\left(B_{m}+D_{m}\right)$.

Thanks to the construction, $\Psi \circ W$ is a mapping from $L^{2}(\Omega)$ to $H^{2}(\Omega) \cap \mathcal{M}$ with

$$
\mathcal{M}:=\left\{v \in H^{2}(\Omega): v \in \overline{D(\beta)} \quad \text { a.e. in } \quad \Omega\right\} .
$$

Testing for $\chi \in \mathcal{M}$ the equation (4.13) by $W(\chi)$, we see that $W(\mathcal{M})$ is a bounded subset of $H^{1}(\Omega)$. Considering (4.13) for two functions $\chi, \chi^{\prime} \in L^{2}(\Omega)$, and testing the difference of the two equations by $W(\chi)-W\left(\chi^{\prime}\right)$, we conclude that $W$ is continuous as function from $L^{2}(\Omega)$ to $H^{1}(\Omega)$. Similar, we get, by using (4.4), that $\Psi(W(\mathcal{M}))$ is a bounded subset of $H^{1}(\Omega)$, and that $\Psi$ is continuous as function from $L^{2}(\Omega)$ to $H^{1}(\Omega)$. Combining this, we see that $\Psi \circ W$ is a continuous function from $L^{2}(\Omega)$ to $L^{2}(\Omega)$, and that $\Psi \circ W\left(\mathcal{M}_{1}\right) \subset \mathcal{M}_{1}$ holds for

$$
\mathcal{M}_{1}:=\left\{v \in \mathcal{M}:\|v\|_{H^{1}(\Omega)} \leqslant \sup \left\{\|w\|_{H^{1}(\Omega)}: w \in \Psi \circ W(\mathcal{M})\right\}\right\} .
$$

Since $\mathcal{M}_{1}$ is a convex, compact subset in $L^{2}(\Omega)$, and $\Psi \circ W$ is continuous, we can apply Schauder's fixed point theorem and get a fixed-point $\chi_{m}$ of $\Psi \circ W$. For $w_{m}:=W\left(\chi_{m}\right) \in H^{2}(\Omega) \subset W^{1,6}(\Omega)$, we get from [31: Theorem 1] that $u_{m}:=\varrho\left(w_{m}\right) \in H^{1}(\Omega)$. Defining now $\theta_{m}:=\psi\left(w_{m}\right)$ and $\xi_{m}$ by (3.4d), we see that the conditions in (3.4) are satisfied. Therefore, the Lemma is proved.

\section{Uniform estimates}

In this section, uniform estimates for solutions to the time-discrete scheme are derived. Assume that (A1)-(A6), and (A9) hold. Let $\beta^{*}:=\partial \phi^{*}$ and $\phi^{*}: \mathbb{R} \rightarrow[0, \infty]$ be either $\phi$ or the function defined by

$$
\phi^{*}(s)= \begin{cases}\phi(s), & \text { if }|s| \leqslant B \\ \infty, & \text { otherwise }\end{cases}
$$

for some $B>\left\|\chi^{0}\right\|_{\infty}$. In the light of (A2), we see that $\phi^{*}$ is a convex, lower semicontinuous function with

$$
0 \leqslant \phi \leqslant \phi^{*} \text { on } \mathbb{R}, \quad 0 \in D\left(\beta^{*}\right), \quad \operatorname{int} D\left(\beta^{*}\right) \neq \emptyset, \quad 0 \in \beta^{*}(0),\left.\quad \phi^{*}\right|_{D\left(\beta^{*}\right)}=\left.\phi\right|_{D\left(\beta^{*}\right)} .
$$

Now, a modified version of the time-discrete scheme is considered, where $\beta$ in (D), i.e. in (3.4b), is replaced by $\beta^{*}$. Let any solution to this scheme be given.

REMARK 5.1 Applying (3.4c), Green's formula, (3.4e), and (2.7), we deduce that

$$
\begin{aligned}
& \int_{\Omega}\left(c_{0} \frac{\theta_{m}-\theta_{m-1}}{h_{m}}+\lambda^{\prime}\left(\chi_{m-1}\right) \frac{\chi_{m}-\chi_{m-1}}{h_{m}}\right) v \mathrm{~d} x+\left(w_{m}, v\right)_{V} \\
&=\int_{\Omega} g_{m} v \mathrm{~d} x+\int_{\Gamma} \mu_{m} v \mathrm{~d} \sigma, \quad \forall v \in H^{1}(\Omega), \quad 1 \leqslant m \leqslant K .
\end{aligned}
$$

In the sequel, $C_{i}$, for $i \in \mathbb{N}$, will always denote generic positive constants, independent of the vector $H$ of time-step sizes, the constant $B$, and the considered solution.

To prepare the a priori estimates, we estimate the data and their approximations. 
Lemma 5.2 There exist positive constants $C_{1}, C_{2}, C_{3}$ such that

$$
\begin{gathered}
\left|\lambda^{\prime}(s)\right|+\left|\sigma^{\prime}(s)\right| \leqslant C_{1}(|s|+1), \quad \forall s \in \mathbb{R} \\
\left|\int_{\Omega} g_{m} v \mathrm{~d} x\right|+\left|\int_{\Gamma} \mu_{m} v \mathrm{~d} \sigma\right| \leqslant C_{2}\|v\|_{V}\left(\left\|g_{m}\right\|_{2}+\left\|\mu_{m}\right\|_{L^{2}(\Gamma)}\right), \quad \forall v \in H^{1}(\Omega), 1 \leqslant m \leqslant K \\
\sum_{m=1}^{K} h_{m}\left(\left\|g_{m}\right\|_{2}^{2}+\left\|\mu_{m}\right\|_{L^{2}(\Gamma)}^{2}\right) \leqslant C_{3}
\end{gathered}
$$

Proof. These estimates follow from (A3), (A4), (3.1), the trace-mapping from $H^{1}(\Omega)$ to $H^{\frac{1}{2}}(\Gamma)$, and the equivalence of the $H^{1}(\Omega)$-norm and $\|\cdot\|_{V}$.

The following Lemmas use ideas from [9-11, 22, 27,35].

LEMMA 5.3 There are two positive constants $C_{4}, C_{5}$ such that

$$
\begin{aligned}
& \max _{0 \leqslant m \leqslant K}\left(\left\|\theta_{m}\right\|_{1}+\left\|\ln \left(\theta_{m}\right)\right\|_{1}+\left\|\chi_{m}\right\|_{H^{1}(\Omega)}^{2}+\left\|\phi\left(\chi_{m}\right)\right\|_{1}\right) \\
& \quad+\sum_{m=1}^{K} h_{m}\left\|u_{m}\right\|_{V}^{2}+\sum_{m=1}^{K} h_{m}\left\|\frac{\chi_{m}-\chi_{m-1}}{h_{m}}\right\|_{2}^{2}+\sum_{m=1}^{K}\left\|\chi_{m}-\chi_{m-1}\right\|_{H^{1}(\Omega)}^{2} \leqslant C_{4}, \\
& \max _{0 \leqslant m \leqslant K}\left(\left\|\lambda^{\prime}\left(\chi_{m}\right)\right\|_{6}+\left\|\sigma^{\prime}\left(\chi_{m}\right)\right\|_{6}\right)+\sum_{m=1}^{K} h_{m}\left\|\lambda^{\prime}\left(\chi_{m-1}\right) \frac{\chi_{m}-\chi_{m-1}}{h_{m}}\right\|_{\frac{3}{2}}^{2} \leqslant C_{5} .
\end{aligned}
$$

Proof. Testing (3.4d) by $\left(\chi_{m}-\chi_{m-1}\right)$, taking the sum from $m=1$ to $m=k$, and using (A6), Green's formula, (3.4e), $\xi_{m} \in \partial \phi^{*}\left(\chi_{m}\right), 0 \leqslant \phi \leqslant \phi^{*}$, the elementary equality

$$
a(a-b)=\frac{1}{2} a^{2}-\frac{1}{2} b^{2}+(a-b)^{2}, \quad \forall a, b \in \mathbb{R},
$$

Hölder's inequality, (3.3), $\phi^{*}\left(\chi^{0}\right)=\phi\left(\chi^{0}\right),(\mathrm{A} 3),(\mathrm{A} 5),(\mathrm{A} 9)$, and Young's inequality, we deduce

$$
\begin{gathered}
\frac{\zeta_{\text {inf }}}{6} \sum_{m=1}^{k} h_{m}\left\|\frac{\chi_{m}-\chi_{m-1}}{h_{m}}\right\|_{2}^{2}+\frac{\varepsilon}{2}\left\|\nabla \chi_{k}\right\|_{2, N}^{2}+\frac{\varepsilon}{2} \sum_{m=1}^{k}\left\|\nabla \chi_{m}-\nabla \chi_{m-1}\right\|_{2, N}^{2}+\left\|\phi\left(\chi_{k}\right)\right\|_{1} \\
\leqslant C_{6}-\sum_{m=1}^{k} \int_{\Omega} \lambda^{\prime}\left(\chi_{m-1}\right)\left(\chi_{m}-\chi_{m-1}\right) u_{m} \mathrm{~d} x+\frac{1}{2 \zeta_{\text {inf }}} \sigma_{\text {factor }}^{\prime} \sum_{m=2}^{k} h_{m}\left\|\phi\left(\chi_{m-1}\right)\right\|_{1} .
\end{gathered}
$$

From $u_{m}=\varrho\left(w_{m}\right),(2.7)$, and (4.5), we get positive constants $C_{7}, C_{8}$, such that

$$
-\left(w_{m}, u_{m}\right)_{V}=-\int_{\Omega} \varrho^{\prime}\left(w_{m}\right)\left|\nabla w_{m}\right|^{2} \mathrm{~d} x-\gamma \int_{\Gamma} w_{m} \varrho\left(w_{m}\right) \mathrm{d} \sigma \geqslant C_{7}\left\|u_{m}\right\|_{V}^{2}-C_{8} .
$$

Because of Taylor's formula and (A3), we have

$$
-\lambda^{\prime}\left(\chi_{m-1}\right)\left(\chi_{m}-\chi_{m-1}\right) \leqslant-\lambda\left(\chi_{m}\right)+\lambda\left(\chi_{m-1}\right)+\frac{1}{2} \lambda_{\text {sup }}^{\prime \prime}\left(\chi_{m}-\chi_{m-1}\right)^{2}, \quad \text { a.e. in } \quad \Omega .
$$

For $1 \leqslant m \leqslant K$ and $\delta:=\min \left(1 /\left(2 \lambda_{\text {factor }}\right), \zeta_{\text {inf }} /\left(6 \lambda_{\text {sup }}^{\prime \prime} T\right)\right)$, we insert $v=h_{m} \delta-h_{m} u_{m}$ in (5.3), use (3.4b), take into account that $\frac{-1}{s}$ is the derivative of the convex function $-\ln (s)$, apply (5.5), 
(5.10), (5.11), and $\delta \lambda_{\text {sup }}^{\prime \prime}<\zeta_{\text {inf }} /(6 T)$. Summing the resulting inequality from $m=1$ to $m=k$, applying Young's inequality, (5.6), (3.4b), (3.3), (A3), and (A5), we conclude that

$$
\begin{aligned}
\delta c_{0}\left\|\theta_{k}\right\|_{1}+c_{0} \int_{\Omega}\left(-\ln \left(\theta_{k}\right)\right) \mathrm{d} x & +\frac{1}{2} C_{7} \sum_{m=1}^{k} h_{m}\left\|u_{m}\right\|_{V}^{2} \leqslant C_{9}+\sum_{m=1}^{k} \int_{\Omega} \lambda^{\prime}\left(\chi_{m-1}\right)\left(\chi_{m}-\chi_{m-1}\right) u_{m} \mathrm{~d} x \\
& +\delta \lambda_{\text {factor }}\left\|\phi\left(\chi_{k}\right)\right\|_{1}+\frac{1}{12 T} \zeta_{\text {inf }} \sum_{m=1}^{k} h_{m}^{2}\left\|\frac{\chi_{m}-\chi_{m-1}}{h_{m}}\right\|_{2}^{2}
\end{aligned}
$$

By elementary analysis we get a positive constant $C_{10}$, such that

$$
\frac{\delta}{2} s+|\ln s| \leqslant \delta s-\ln s+C_{10}, \quad \forall s>0 .
$$

We use this inequality to estimate the first two summands in (5.12) from below. Adding the resulting estimate to (5.9), and using $\delta \lambda_{\text {factor }} \leqslant 1 / 2$, the discrete version of Gronwall's lemma, (A9), (3.3), and (A5), we conclude that (5.7) is satisfied.

Thanks to Hölder's inequality, we have

$$
\left\|\lambda^{\prime}\left(\chi_{m-1}\right) \frac{\chi_{m}-\chi_{m-1}}{h_{m}}\right\|_{\frac{3}{2}} \leqslant\left\|\lambda^{\prime}\left(\chi_{m-1}\right)\right\|_{6}\left\|\frac{\chi_{m}-\chi_{m-1}}{h_{m}}\right\|_{2}, \quad \forall 1 \leqslant m \leqslant K .
$$

Because of (5.7) and Sobolev's embedding theorem, we have a uniform upper bound for $\left\|\chi_{m}\right\|_{6}$. In the light of (5.4), (5.7), and (5.13), we observe that (5.8) holds.

LEMMA 5.4 There is a positive constant $C_{11}$ such that

$$
\max _{0 \leqslant m \leqslant K}\|\tilde{\alpha}(\theta)\|_{L^{1}(\Omega)}+\sum_{m=1}^{K} h_{m}\left\|w_{m}\right\|_{V}^{2} \leqslant C_{11} .
$$

Proof. For $1 \leqslant m \leqslant K$, we insert $v=h_{m} w_{m}$ in (5.3), use (3.4b), take into account that $\alpha$ is the derivative of the convex function $\tilde{\alpha}$, and apply (5.5), Hölder's inequality, the continuous embedding of $V$ in $L^{6}(\Omega)$, and Young's inequality, to conclude that

$$
\begin{aligned}
& c_{0} \int_{\Omega} \tilde{\alpha}\left(\theta_{m}\right) \mathrm{d} x-c_{0} \int_{\Omega} \tilde{\alpha}\left(\theta_{m-1}\right) \mathrm{d} x+\frac{1}{2} h_{m}\left\|w_{m}\right\|_{V}^{2} \\
& \quad \leqslant h_{m} C_{12}\left(\left\|\lambda^{\prime}\left(\chi_{m-1}\right) \frac{\chi_{m}-\chi_{m-1}}{h_{m}}\right\|_{6 / 5}^{2}+\left\|g_{m}\right\|_{2}^{2}+\left\|\mu_{m}\right\|_{L^{2}(\Gamma)}^{2}\right) .
\end{aligned}
$$

Summing up this equation from $m=1$ to $m=K$ and using (5.6), (5.8), and (4.1), we see that (5.14) is satisfied.

LEMMA 5.5 There is a positive constant $C_{13}$ such that

$$
\sum_{m=1}^{K} h_{m}\left\|\frac{\theta_{m}-\theta_{m-1}}{h_{m}}\right\|_{V^{*}}^{2} \leqslant C_{13}
$$


Proof. In view of the terms in (5.3) and the estimates (5.5)-(5.8), (5.14), and the continuity of the embedding of $L^{\frac{6}{5}}(\Omega)$ in $H^{1}(\Omega)^{*}$ and in $V^{*}$, we conclude that (5.15) is satisfied.

LEMmA 5.6 There exists a positive constant $C_{14}$ such that

$$
\sum_{m=1}^{K} h_{m}\left\|\xi_{m}\right\|_{2}^{2}+\sum_{m=1}^{K} h_{m}\left\|\chi_{m}\right\|_{H^{2}(\Omega)}^{2} \leqslant C_{14}
$$

Proof. We use a classical elliptic estimate (see, for example, [2: Remark 9.3d]), (5.7), and (3.4e), and compare the terms in $(3.4 \mathrm{~d})$, to derive that

$$
\left\|\chi_{m}\right\|_{H^{2}(\Omega)} \leqslant C_{15}+C_{16}\left\|\Delta \chi_{m}\right\|_{2} \leqslant C_{15}+C_{16} \frac{1}{\varepsilon}\left\|f_{m}^{*}-\xi_{m}\right\|_{2},
$$

with $f_{m}^{*} \in L^{2}(\Omega)$ defined by

$$
f_{m}^{*}:=-\lambda^{\prime}\left(\chi_{m-1}\right) u_{m}-\zeta\left(\nabla \chi_{m-1}\right) \frac{\chi_{m}-\chi_{m-1}}{h_{m}}+\sigma^{\prime \prime}\left(\chi_{m-1}\right)\left(\chi_{m}-\chi_{m-1}\right)+\sigma^{\prime}\left(\chi_{m-1}\right) .
$$

Testing formally (3.4d) by $\xi_{m}$ and using Green's formula, (3.4e), (3.4b), Young's inequality, and (5.18), we observe that

$$
\left\|\xi_{m}\right\|_{2} \leqslant\left\|f_{m}^{*}\right\|_{2}
$$

For a precise derivation of this inequality, one has to consider for $n \in \mathbb{N}$ a nonlinear elliptic problem arising by considering (3.4d) with $\chi_{m}$ and $\xi_{m}$ replaced by $\chi_{m, n}$ and $\beta_{\frac{1}{n}}\left(\chi_{m, n}\right)$, respectively, where $\beta_{\frac{1}{n}}$ is the Yosida approximation of $\beta$. This equation is tested by $\chi_{m, n}$ and $\beta_{\frac{1}{n}}\left(\chi_{m, n}\right)$. Now, a passage to the limit and [3: Chapter II Proposition 1.1(iv)] lead to (5.19).

Because of (5.18), the discrete Schwarz's inequality, Hölder's inequality, (A6), and (A3), we have

$$
\left\|f_{m}^{*}\right\|_{2}^{2} \leqslant 3\left(\left\|\lambda^{\prime}\left(\chi_{m-1}\right)\right\|_{4}^{2}\left\|u_{m}\right\|_{4}^{2}+\left(\zeta_{\text {sup }}+\sigma_{\text {sup }}^{\prime \prime} h_{m}\right)^{2}\left\|\frac{\chi_{m}-\chi_{m-1}}{h_{m}}\right\|_{2}^{2}+\left\|\sigma^{\prime}\left(\chi_{m-1}\right)\right\|_{2}^{2}\right) .
$$

Thanks to Sobolev's embedding theorem, the equivalences of the norms $\|\cdot\|_{V}$ and $\|\cdot\|_{H^{1}(\Omega)}$, and (5.7), we have a uniform upper bound for $\left\|u_{m}\right\|_{4}$. Hence, in the light of (5.20), (5.8), (5.17), and (5.19), we see that (5.16) is satisfied.

LEMMA 5.7 We have

$$
\sum_{m=1}^{K} h_{m}\left\|\chi_{m}-\chi_{m-1}\right\|_{H^{1}(\Omega)}^{6} \leqslant C_{17} h_{\max }(H) .
$$

Proof. Applying that $a^{6}=a^{4} a^{2}$ holds for all $a \in \mathbb{R}$ and recalling (5.7), we conclude that (5.21) is satisfied.

LEMMA 5.8 If (A7) and (A8) hold, there exists a positive constant $C_{18}$ such that

$$
\sum_{m=1}^{K} h_{m}\left\|\nabla\left(\ln \left(\theta_{m}\right)\right)\right\|_{2, N}^{2}+\max _{0 \leqslant m \leqslant K}\left\|\theta_{m}\right\|_{2} \leqslant C_{18} .
$$


Proof. We multiply (3.4c) by $h_{m}$, sum up the resulting equation for $m=1$ to $m=i$ and apply (3.3), to derive that

$$
c_{0} \theta_{i}-\sum_{m=1}^{i} h_{m} \Delta w_{m}=c_{0} \theta^{0}+\sum_{m=1}^{i} h_{m}\left(g_{m}-\lambda^{\prime}\left(\chi_{m-1}\right) \frac{\chi_{m}-\chi_{m-1}}{h_{m}}\right), \quad \text { a.e. in } \quad \Omega .
$$

Because of (A7), (3.4b), the continuity of $w_{i}$ on $\bar{\Omega}, u_{i}>0$ a.e. in $\Omega$, we see that $w_{i}=$ $-\alpha_{\text {low }} u_{i}=-\alpha_{\text {low }} / \theta_{i}$ a.e. in $\Omega$, and that $u_{i} \geqslant 0$ in $\Gamma$. Moreover, $\mu_{i} \leqslant 0$ a.e. in $\Gamma$ because of (3.1) and (A8). Hence, by applying Green's formula, (3.4b), and (3.4e), we get, at least formally,

$$
-\int_{\Omega} \theta_{i} \Delta w_{i} \mathrm{~d} x=\alpha_{\text {low }} \int_{\Omega} \theta_{i} \Delta u_{i} \mathrm{~d} x \geqslant \alpha_{\text {low }}\left\|u_{i} \nabla \theta_{i}\right\|_{2, N}^{2}-C_{19}=\alpha_{\text {low }}\left\|\nabla \ln \left(\theta_{i}\right)\right\|_{2, N}^{2}-C_{19} .
$$

For a precise derivation of this inequality, one has to perform this computation with $\theta_{i}$ replaced by the approximation $\theta_{i, l} \in H^{1}(\Omega)$ defined by

$$
\theta_{i, l}:=\left(u_{i}+\frac{1}{l}\right)^{-1}, \quad \text { a.e. in } \quad \Omega, \quad \forall l \in \mathbb{N},
$$

and consider afterwards the limit for $l \rightarrow \infty$, using the Lebesgue-dominated convergence theorem. Indeed, we have strong convergences for $\theta_{i, l}$ in $L^{2}(\Omega)$, so that $\theta_{i, l} \nabla u_{i} \longrightarrow \theta_{i} \nabla u_{i}$ strongly in $\left(L^{1}(\Omega)\right)^{3}$ and weakly in $\left(L^{2}(\Omega)\right)^{3}$.

Because of (5.24), we can test (5.23) by $h_{i} \cdot \Delta w_{i}$, take the sum from $i=1$ to $i=k$ over the resulting equation, and utilize the formulae [27: (AP.2), (AP.3)] for the generated double sums. Applying afterwards Schwarz's inequality, Young's inequality, (A5), (5.6), (A8), (5.7), and (A9), we observe that

$$
\begin{aligned}
c_{0} \alpha_{\text {low }} & \sum_{i=1}^{k} h_{i}\left\|\nabla \ln \left(\theta_{i}\right)\right\|_{2, N}^{2}+\frac{1}{2}\left\|\sum_{i=1}^{k} h_{i} \Delta w_{i}\right\|_{2}^{2}+\frac{1}{2} \sum_{i=1}^{k} h_{i}^{2}\left\|\Delta w_{i}\right\|_{2}^{2} \\
\leqslant & C_{20}+\int_{\Omega}\left(\left(c_{0} \theta^{0}+\sum_{i=1}^{k} h_{i}\left(g_{i}-\lambda^{\prime}\left(\chi_{i-1}\right) \frac{\chi_{i}-\chi_{i-1}}{h_{i}}\right)\right) \sum_{i=1}^{k} h_{i} \Delta w_{i}\right) \mathrm{d} x \\
& -\sum_{i=1}^{k-1} h_{i+1} \int_{\Omega}\left(\left(g_{i+1}-\lambda^{\prime}\left(\chi_{i}\right) \frac{\chi_{i+1}-\chi_{i}}{h_{i+1}}\right) \sum_{m=1}^{i} h_{m} \Delta w_{m}\right) \mathrm{d} x . \\
\leqslant & C_{21}+C_{22} \sum_{i=1}^{k-1} h_{i}\left\|\sum_{m=1}^{i} h_{m} \Delta w_{m}\right\|_{2}^{2}+\frac{1}{4}\left\|\sum_{i=1}^{k} h_{i} \Delta w_{i}\right\|_{2}^{2} .
\end{aligned}
$$

Thanks to the discrete version of Gronwall's lemma, there is a uniform upper bound for the left-hand side of (5.25). Comparing now the terms in (5.23) and using (A5), (5.22), Schwarz's inequality, (5.6), and (3.3), we see that (5.22) holds.

\section{The proof of Theorem 3.2}

Proof. Assume that (A1)-(A6) and (A9) are satisfied. 
For any $B>\left\|\chi^{0}\right\|_{\infty}$, we can consider $\phi^{*}$ as in (5.1), $\beta^{*}$, and the corresponding modified version of the time-discrete scheme as in the last section. Lemma 4.2 yields that there exists a solution $\left(\theta_{m}^{B}, w_{m}^{B}, u_{m}^{B}, \chi_{m}^{B}, \xi_{m}^{B}\right)_{m=0}^{K}$ to this modified version of the scheme, and the estimates derived in the last section hold for this solution. Now, because of (5.16) and Sobolev's embedding theorem, there is some positive constant $C^{\prime}$, independent of $B$, such that

$$
\left(\min _{1 \leqslant m \leqslant K} h_{m}\right) \max _{1 \leqslant m \leqslant K}\left\|\chi_{m}^{B}\right\|_{C(\bar{\Omega})}^{2} \leqslant \sum_{m=1}^{K} h_{m}\left\|\chi_{m}^{B}\right\|_{C(\bar{\Omega})}^{2} \leqslant C^{\prime} .
$$

Hence, we see that for $B$ sufficiently large the solution to the modified version of the scheme is also a solution to the unmodified version of the scheme. This proves part (1) of Theorem 3.2.

To prove part (2) of Theorem 3.2, we assume that (A7) holds and that we have some $m \in$ $\{1, \ldots, K\}, \theta_{m-1} \in L^{2}(\Omega), \chi_{m-1} \in H^{2}(\Omega)$, and two solutions $\left(\theta_{m}^{(1)}, w_{m}^{(1)}, u_{m}^{(1)}, \chi_{m}^{(1)}, \xi_{m}^{(1)}\right)$, $\left(\theta_{m}^{(2)}, w_{m}^{(2)}, u_{m}^{(2)}, \chi_{m}^{(2)}, \xi_{m}^{(2)}\right)$ to the system (3.4) in (D).

Let $\theta_{m}^{*}:=\theta_{m}^{(1)}-\theta_{m}^{(2)}$, and $w_{m}^{*}, u_{m}^{*}, \chi_{m}^{*}, \xi_{m}^{*}$ be defined analogously. First, we consider (5.3) with $v=h_{m} w_{m}^{*}$ for both solutions, and compute the difference of the resulting equations. Then, (3.4d) is considered for both solutions, and the difference of the resulting equations is tested by $\alpha_{\text {low }} \chi_{m}^{*}$. Adding the resulting equations, and applying (3.4b), the monotonicity of $\alpha$ and $\beta,(3.4 \mathrm{e}),(\mathrm{A} 6)$, (A3), and (A9), we get

$$
\begin{aligned}
c_{0}\left\|\theta_{m}^{*} w_{m}^{*}\right\|_{1}+h_{m}\left\|w_{m}^{*}\right\|_{V}^{2}+\frac{2}{3} \frac{1}{h_{m}} \zeta_{\text {inf }} \alpha_{\text {low }}\left\|\chi_{m}^{*}\right\|_{2}^{2} & +\alpha_{\text {low }} \varepsilon\left\|\nabla \chi_{m}^{*}\right\|_{2, N}^{2}+\alpha_{\text {low }}\left\|\chi_{m}^{*} \xi_{m}^{*}\right\|_{1} \\
& \leqslant-\int_{\Omega} \lambda^{\prime}\left(\chi_{m-1}\right)\left(w_{m}^{*}+\alpha_{\text {low }} u_{m}^{*}\right) \chi_{m}^{*} \mathrm{~d} x .
\end{aligned}
$$

Because of (A7) and (3.4b), the right-hand side of (6.2) is equal to zero, and therefore $\chi_{m}^{*}=0$ and $w_{m}^{*}=0$. Using (3.4b) and that $\alpha$ is injective, we see that $\theta_{m}^{(1)}=\theta_{m}^{(2)}$ and $u_{m}^{(1)}=u_{m}^{(2)}$. Now, (3.4d) yields that $\xi_{m}^{(1)}=\xi_{m}^{(2)}$. This completes the proof of Theorem 3.2.

\section{Convergence of the time-discrete scheme}

In this section, Theorem 3.4 is proved. We assume that (A2)-(A6) hold, and that we have a sequence $\left\{H^{(n)}\right\}_{n \in \mathbb{N}}$ of vectors $H^{(n)}=\left(h_{1}^{(n)}, h_{2}^{(n)}, \ldots, h_{K^{(n)}}^{(n)}\right)$ of time-step sizes with (A9) and $h_{\max }\left(H^{(n)}\right) \underset{n \rightarrow \infty}{\longrightarrow} 0$.

Hence, Theorem 3.2 yields that for every $H^{(n)}$ there exists a solution to the time-discrete scheme (D). Let $\left(\widehat{\theta}^{(n)}, \bar{w}^{(n)}, \bar{u}^{(n)}, \widehat{\chi}^{(n)}, \bar{\xi}^{(n)}\right)$ be the corresponding approximations derived from the solution to (D) as in Section 3. Moreover, we define the piecewise constant functions $\bar{\theta}^{(n)}, \bar{\chi}^{(n)}$, $\bar{\mu}^{(n)}, \bar{g}^{(n)}$, analogously to $\bar{w}^{(n)}$, while $\underline{\chi}^{(n)} \in L^{\infty}\left(0, T ; H^{2}(\Omega)\right)$ is defined by

$$
\underline{\chi}^{(n)}(t)=\widehat{\chi}^{(n)}\left(t_{m-1}^{(n)}\right)=\chi_{m-1}^{(n)}, \quad \forall t \in\left[t_{m-1}^{(n)}, t_{m}^{(n)}\right), 1 \leqslant m \leqslant K^{(n)},
$$

with $t_{0}^{(n)}:=0$ and $t_{m}^{(n)}:=\sum_{i=1}^{m} h_{i}$, for $1 \leqslant m \leqslant K^{(n)}$. 
Then, by the definition of the approximations, (3.3), (3.4), Lemma 2.2, (A5), and (5.3), we have

$$
\begin{gathered}
\widehat{\theta}^{(n)} \in H^{1}\left(0, T ; L^{2}(\Omega)\right), \quad \bar{w}^{(n)} \in L^{\infty}\left(0, T ; H^{2}(\Omega)\right), \quad \bar{u}^{(n)} \in L^{\infty}\left(0, T ; H^{1}(\Omega)\right), \\
\widehat{\chi}^{(n)} \in H^{1}\left(0, T ; H^{2}(\Omega)\right), \quad \bar{\chi}^{(n)}, \underline{\chi}^{(n)} \in L^{\infty}\left(0, T ; H^{2}(\Omega)\right), \\
\bar{\xi}^{(n)} \in L^{\infty}\left(0, T ; L^{2}(\Omega)\right), \\
F \bar{w}^{(n)}(t) \in \partial_{*} j\left(\bar{\theta}^{(n)}(t)\right), \quad-F \bar{u}^{(n)}(t) \in \partial_{*} \tilde{J}\left(\bar{\theta}^{(n)}(t)\right) \quad \text { in } \quad V^{*}, \quad \text { for a.e. } t \in(0, T), \\
\bar{\chi}^{(n)}, \widehat{\chi}^{(n)}, \underline{\chi}^{(n)} \in D(\beta), \quad \bar{\xi}^{(n)} \in \beta\left(\bar{\chi}^{(n)}\right), \quad \text { a.e. in } \quad \Omega_{T}, \\
c_{0}\left\langle\widehat{\theta}_{t}^{(n)}(t), v\right\rangle_{V^{*} \times V}+\int_{\Omega} \lambda^{\prime}\left(\underline{\chi}^{(n)}(t)\right) \widehat{\chi}_{t}^{(n)}(t) v \mathrm{~d} x+\left(\bar{w}^{(n)}(t), v\right)_{V} \\
=\int_{\Omega} \bar{g}^{(n)}(t) v \mathrm{~d} x+\int_{\Gamma} \bar{\mu}^{(n)}(t) v \mathrm{~d} \sigma, \quad \forall v \in H^{1}(\Omega), \quad \text { for a.e. } \quad t \in(0, T), \\
\zeta\left(\nabla \underline{\chi}^{(n)}\right) \widehat{\chi}_{t}^{(n)}-\varepsilon \Delta \bar{\chi}^{(n)}+\bar{\xi}^{(n)}-\sigma^{\prime \prime}\left(\underline{\chi}^{(n)}\right)\left(\bar{\chi}^{(n)}-\underline{\chi}^{(n)}\right)-\sigma^{\prime}\left(\underline{\chi}^{(n)}\right) \\
=-\lambda^{\prime}\left(\underline{\chi}^{(n)}\right) \bar{u}^{(n)}, \quad \text { a.e. in } \quad \Omega_{T}, \\
\frac{\partial \bar{\chi}^{(n)}}{\partial n}=0, \quad \text { a.e. in } \Gamma_{T}, \\
\widehat{\theta}^{(n)}(\cdot, 0)=\theta^{0}, \quad \hat{\chi}^{(n)}(\cdot, 0)=\chi^{0}, \quad \text { a.e. in } \Omega .
\end{gathered}
$$

From (A4) and (3.1), we obtain by a density argument

$$
\left\|g-\bar{g}^{(n)}\right\|_{L^{2}\left(0, T ; L^{2}(\Omega)\right)}+\left\|\mu-\bar{\mu}^{(n)}\right\|_{L^{2}\left(0, T ; H^{\frac{1}{2}}(\Gamma)\right)} \underset{n \rightarrow \infty}{\longrightarrow} 0 .
$$

In the sequel, $C_{i}$, for $i \in \mathbb{N}$, will always denote positive generic constants, independent of $n$. We find, from (5.7), (5.14), (5.15), (5.16), (3.3), and (A5):

$$
\begin{aligned}
& \left\|\widehat{\theta}^{(n)}\right\|_{H^{1}\left(0, T ; V^{*}\right) \cap L^{\infty}\left(0, T ; L^{1}(\Omega)\right)}+\left\|\bar{\theta}^{(n)}\right\|_{L^{\infty}\left(0, T ; V^{*} \cap L^{1}(\Omega)\right)} \\
& \quad+\left\|\bar{w}^{(n)}\right\|_{L^{2}(0, T ; V)}+\left\|\bar{u}^{(n)}\right\|_{L^{2}(0, T ; V)} \leqslant C_{1}, \\
& \quad\left\|\widehat{\chi}^{(n)}\right\|_{H^{1}\left(0, T ; L^{2}(\Omega)\right) \cap C\left([0, T] ; H^{1}(\Omega)\right) \cap L^{2}\left(0, T ; H^{2}(\Omega)\right)}+\left\|\bar{\chi}^{(n)}\right\|_{L^{\infty}\left(0, T ; H^{1}(\Omega)\right) \cap L^{2}\left(0, T ; H^{2}(\Omega)\right)} \\
& \quad+\left\|\underline{\chi}^{(n)}\right\|_{L^{\infty}\left(0, T ; H^{1}(\Omega)\right) \cap L^{2}\left(0, T ; H^{2}(\Omega)\right)}+\left\|\bar{\xi}^{(n)}\right\|_{L^{2}\left(0, T ; L^{2}(\Omega)\right)} \leqslant C_{2} .
\end{aligned}
$$

The difference between the different approximations can be estimated, by using (5.7), (5.15), and (5.21):

$$
\begin{aligned}
\left\|\widehat{\chi}^{(n)}-\bar{\chi}^{(n)}\right\|_{L^{2}\left(0, T ; L^{2}(\Omega)\right)}+\| \underline{\chi}^{(n)}- & \bar{\chi}^{(n)}\left\|_{L^{2}\left(0, T ; L^{2}(\Omega)\right)}+\right\| \widehat{\chi}^{(n)}-\underline{\chi}^{(n)} \|_{L^{6}\left(0, T ; H^{1}(\Omega)\right)}^{6} \\
& +\left\|\widehat{\theta}^{(n)}-\bar{\theta}^{(n)}\right\|_{L^{2}\left(0, T ; V^{*}\right)} \leqslant C_{3} h_{\max }\left(H^{(n)}\right) \underset{n \rightarrow \infty}{\longrightarrow} 0 .
\end{aligned}
$$

Thanks to the estimates (7.4), (7.5), compactness arguments and (7.6), we get a subsequence $\left\{n_{k}\right\}_{k \in \mathbb{N}}$, a function $\theta:(0, T) \rightarrow V^{*}$, and functions $w, u, \chi, \xi: \Omega_{T} \rightarrow \mathbb{R}$, such that we have the convergences (3.6)-(3.11) and

$$
\begin{aligned}
& \bar{\theta}^{\left(n_{k}\right)} \underset{k \rightarrow \infty}{\longrightarrow} \theta, \quad \text { weakly-star in } \quad L^{\infty}\left(0, T ; V^{*}\right), \\
& \bar{\chi}^{\left(n_{k}\right)} \underset{k \rightarrow \infty}{\longrightarrow} \chi, \quad \text { weakly in } \quad L^{2}\left(0, T ; H^{2}(\Omega)\right) .
\end{aligned}
$$


Now, we will show that $(\theta, w, u, \chi, \xi)$ is a solution to the PF system. Thanks to the convergences (3.6)-(3.11), we see that (2.14a) and (2.14b) are satisfied.

Because of (7.5), the Aubin Lemma as in [34: Corollary 8] implies that the sequence $\left\{\widehat{\chi}^{\left(n_{k}\right)}\right\}_{k \in \mathbb{N}}$ is relatively compact in $L^{6}\left(0, T ; H^{1}(\Omega)\right)$. Therefore, by (3.9),

$$
\widehat{\chi}^{\left(n_{k}\right)} \underset{k \rightarrow \infty}{\longrightarrow} \chi, \quad \text { strongly in } \quad L^{6}\left(0, T ; H^{1}(\Omega)\right) .
$$

Recalling (7.6) and the continuous embedding of $H^{1}(\Omega)$ in $L^{6}(\Omega)$, we deduce that

$$
\begin{gathered}
\underline{\chi}^{\left(n_{k}\right)} \underset{k \rightarrow \infty}{\longrightarrow} \chi, \quad \begin{array}{l}
\text { strongly in } \\
\text { strongly in }
\end{array} L^{6}\left(0, T ; H^{1}(\Omega)\right),
\end{gathered}
$$

Thus, we can extract a subsequence $\left\{n_{k_{l}}\right\}_{l \in \mathbb{N}}$ from $\left\{n_{k}\right\}_{k \in \mathbb{N}}$ such that we have a.e. convergence for $\underline{\chi}^{\left(n_{k}\right)}$ and $\nabla \underline{\chi}^{\left(n_{k}\right)}$ to $\chi$ and $\nabla \chi$, respectively. Now, we can assume without losing generality that already $\left\{n_{k}\right\}_{k \in \mathbb{N}}$ has been chosen in such a way that these convergences are satisfied. Hence, by applying the generalized Lebesgue-dominated convergence theorem (see, for example, [1: A-1.26]), (5.4), (7.11), and (A6), we conclude that

$$
\begin{aligned}
\lambda^{\prime}\left(\underline{\chi}^{\left(n_{k}\right)}\right) \underset{k \rightarrow \infty}{\longrightarrow} \lambda^{\prime}(\chi), & \text { strongly in } L^{6}\left(\Omega_{T}\right), \\
\sigma^{\prime}\left(\underline{\chi}^{\left(n_{k}\right)}\right) \underset{k \rightarrow \infty}{\longrightarrow} \sigma^{\prime}(\chi), & \text { strongly in } L^{6}\left(\Omega_{T}\right), \\
\zeta\left(\nabla \underline{\chi}^{\left(n_{k}\right)}\right) \underset{k \rightarrow \infty}{\longrightarrow} \zeta(\nabla \chi), & \text { strongly in } L^{p}\left(\Omega_{T}\right), \quad \forall 1 \leqslant p<\infty .
\end{aligned}
$$

Combining this with (3.9) and Hölder's inequality, we observe

$$
\lambda^{\prime}\left(\underline{\chi}^{\left(n_{k}\right)}\right) \widehat{\chi}_{t}^{\left(n_{k}\right)} \underset{k \rightarrow \infty}{\longrightarrow} \lambda^{\prime}(\chi) \chi_{t}, \quad \text { weakly in } \quad L^{\frac{3}{2}}\left(0, T ; L^{\frac{3}{2}}(\Omega)\right) .
$$

From (3.8) it follows by Sobolev's embedding theorem that $\bar{u}^{\left(n_{k}\right)}$ converges to $u$ weakly in $L^{2}\left(0, T ; L^{6}(\Omega)\right)$. Thus, we get from (7.12) by using Hölder's inequality

$$
\lambda^{\prime}\left(\underline{\chi}^{\left(n_{k}\right)}\right) \bar{u}^{\left(n_{k}\right)} \underset{k \rightarrow \infty}{\longrightarrow} \lambda^{\prime}(\chi) u, \quad \text { weakly in } \quad L^{\frac{3}{2}}\left(0, T ; L^{3}(\Omega)\right) .
$$

Thanks to (A3), (7.6), and (7.13), we have

$$
-\sigma^{\prime \prime}\left(\underline{\chi}^{\left(n_{k}\right)}\right)\left(\bar{\chi}^{\left(n_{k}\right)}-\underline{\chi}^{\left(n_{k}\right)}\right)-\sigma^{\prime}\left(\underline{\chi}^{\left(n_{k}\right)}\right) \underset{k \rightarrow \infty}{\longrightarrow}-\sigma^{\prime}(\chi), \quad \text { strongly in } \quad L^{2}\left(0, T ; L^{2}(\Omega)\right) .
$$

Applying (7.2f), (3.6), (7.15), (3.7), (7.3), and (2.7), we conclude that (2.14e) is satisfied. Recalling (7.2g), (7.14), (3.9), Hölder's inequality, (7.8), (3.11), (7.17), and (7.16), we conclude that (2.14f) is satisfied. Moreover, (7.2h) and (7.8) produce $(2.14 \mathrm{~g})$, and $(2.14 \mathrm{~h})$ is satisfied because of (7.2i), (3.6), and (3.9).

Using (7.9), (7.6), and (3.11), we observe that

$$
\int_{0}^{T} \int_{\Omega} \bar{\chi}^{\left(n_{k}\right)} \bar{\xi}^{\left(n_{k}\right)} \mathrm{d} x \mathrm{~d} t \underset{k \rightarrow \infty}{\longrightarrow} \int_{0}^{T} \int_{\Omega} \chi \xi \mathrm{d} x \mathrm{~d} t .
$$


Now, we combine this with (7.2e), (7.8), (3.11), and [3: Chapter II, Lemma 1.3], to show that (2.14d) is satisfied.

Hence, it remains only to show that (2.14c) is satisfied to prove that $(\theta, w, u, \chi, \xi)$ is a solution to the PF system. This is done by following and extending the calculations in [23: (4.10)-(4.16)]. Inserting $v=F^{-1} \widehat{\theta}^{(n)}(t)$ in (7.2f), we get, by (2.9) and (2.10):

$$
\begin{aligned}
\frac{1}{2} c_{0} \frac{\mathrm{d}}{\mathrm{d} t}\left\|\widehat{\theta}^{(n)}(t)\right\|_{V^{*}}^{2}+\int_{\Omega} \lambda^{\prime}\left(\underline{\chi}^{(n)}(t)\right) \widehat{\chi}_{t}^{(n)}(t) F^{-1} \widehat{\theta}^{(n)}(t) \mathrm{d} x+\left(F \bar{w}^{(n)}(t), \widehat{\theta}^{(n)}(t)\right)_{*} \\
=\int_{\Omega} \bar{g}^{(n)}(t) F^{-1} \widehat{\theta}^{(n)}(t) \mathrm{d} x+\int_{\Gamma} \bar{\mu}^{(n)}(t) F^{-1} \widehat{\theta}^{(n)}(t) \mathrm{d} \sigma, \quad \text { a.e. in } \quad(0, T) .
\end{aligned}
$$

Since $V$ is compactly embedded in $L^{3}(\Omega)$, the Aubin Lemma yields that $H^{1}(0, T ; V)$ is compactly embedded in $C\left([0, T] ; L^{3}(\Omega)\right)$. Hence, the continuity of the map $F^{-1}: V^{*} \rightarrow V$ and (3.6) yield that

$$
F^{-1} \widehat{\theta}^{\left(n_{k}\right)} \underset{k \rightarrow \infty}{\longrightarrow} F^{-1} \theta, \quad \text { strongly in } \quad C\left([0, T] ; L^{3}(\Omega)\right) .
$$

Hence, integrating (7.18) from 0 to $s \in[0, T]$ and using (7.15), (7.19), Hölder's inequality, and (7.3) produce

$$
\begin{aligned}
& \frac{1}{2} c_{0}\left\|\widehat{\theta}^{\left(n_{k}\right)}(s)\right\|_{V *}^{2}+\int_{0}^{s}\left(F \bar{w}^{\left(n_{k}\right)}(t), \widehat{\theta}^{\left(n_{k}\right)}(t)\right)_{*} \mathrm{~d} t \underset{k \rightarrow \infty}{\longrightarrow} \frac{1}{2} c_{0}\|\theta(0)\|_{V^{*}}^{2} \\
& \quad-\int_{0}^{s} \int_{\Omega} \lambda^{\prime}(\chi(t)) \chi_{t}(t) F^{-1} \theta(t) \mathrm{d} x \mathrm{~d} t+\int_{0}^{s}\left(\int_{\Omega} g(t) F^{-1} \theta(t) \mathrm{d} x+\int_{\Gamma} \mu(t) F^{-1} \theta(t) \mathrm{d} \sigma\right) \mathrm{d} t .
\end{aligned}
$$

Inserting $v=F^{-1}(\theta(t))$ in (2.14e), integrating the resulting equation from 0 to $s$, and applying (2.9), (2.10), (7.20), (7.6), and (7.4), we have proved that

$$
\frac{1}{2} c_{0}\left\|\hat{\theta}^{\left(n_{k}\right)}(s)\right\|_{V^{*}}^{2}+\int_{0}^{s}\left(F \bar{w}^{\left(n_{k}\right)}(t), \bar{\theta}^{\left(n_{k}\right)}(t)\right)_{*} \mathrm{~d} t \underset{k \rightarrow \infty}{\longrightarrow} \frac{1}{2} c_{0}\|\theta(s)\|_{V^{*}}^{2}+\int_{0}^{s}(F w(t), \theta(t))_{*} \mathrm{~d} t .
$$

Hence, (3.6) yields that

$$
\limsup _{k \rightarrow \infty} \int_{0}^{s}\left(F \bar{w}^{\left(n_{k}\right)}(t), \bar{\theta}^{\left(n_{k}\right)}(t)\right)_{*} \mathrm{~d} t \leqslant \int_{0}^{s}(F w(t), \theta(t))_{*} \mathrm{~d} t, \quad \forall 0 \leqslant s \leqslant T .
$$

Combining this with (7.2d), (7.7), (3.7), (7.22) for $s=T$, and [3: Chapter II, Lemma 1.3], we deduce that

$$
\begin{aligned}
& F w(t) \in \partial_{*} j(\theta(t)) \quad \text { in } \quad V^{*}, \quad \text { for a.e. } \quad t \in(0, T), \\
& 0 \leqslant \liminf _{k \rightarrow \infty} \int_{0}^{s}\left(F \bar{w}^{\left(n_{k}\right)}(t)-F w(t), \bar{\theta}^{\left(n_{k}\right)}(t)-\theta(t)\right)_{*} \mathrm{~d} t \\
& \quad=\liminf _{k \rightarrow \infty} \int_{0}^{s}\left(F \bar{w}^{\left(n_{k}\right)}(t), \bar{\theta}^{\left(n_{k}\right)}(t)\right)_{*} \mathrm{~d} t-\int_{0}^{s}(F w(t), \theta(t))_{*} \mathrm{~d} t, \quad \forall 0 \leqslant s \leqslant T .
\end{aligned}
$$


Hence, (7.21) and (7.22) lead to

$$
\left\|\widehat{\theta}^{\left(n_{k}\right)}(s)\right\|_{V^{*}}^{2} \underset{k \rightarrow \infty}{\longrightarrow}\|\theta(s)\|_{V^{*}}^{2}, \quad \forall s \in[0, T] .
$$

Therefore, we get from (3.6)

$$
\widehat{\theta}^{\left(n_{k}\right)}(s) \underset{k \rightarrow \infty}{\longrightarrow} \theta(s), \quad \text { strongly in } V^{*}, \quad \forall s \in[0, T] .
$$

Since the sequence $\left\{\widehat{\theta}^{\left(n_{k}\right)}\right\}_{k \in \mathbb{N}}$ of continuous functions from $[0, T]$ to $V^{*}$ is uniformly equicontinuous by (7.4), we now recall Ascoli's Theorem and the equivalence of the space $V^{*}$ and $H^{1}(\Omega)^{*}$, to show that (3.5) is satisfied. Applying (3.8), we observe that

$$
\lim _{k \rightarrow \infty} \int_{0}^{T}\left(-F \bar{u}^{\left(n_{k}\right)}(t), \bar{\theta}^{\left(n_{k}\right)}(t)\right)_{*} \mathrm{~d} t=\int_{0}^{T}(-F u(t), \theta(t))_{*} \mathrm{~d} t .
$$

Combining this with (7.2d), (3.5), (3.8), [3: Chapter II, Lemma 1.3], and the already proved compatibility (7.23) between $w$ and $\theta$, we see that (2.14c) is satisfied. Hence, we have shown that $(\theta, w, u, \chi, \xi)$ is a solution to the PF system, and the first assertion in Theorem 3.4 is proved.

To prove the second assertion, we assume that (A7) and (A8) are satisfied. Therefore, (5.22) yields that

$$
\left\|\widehat{\theta}^{(n)}\right\|_{L^{\infty}\left(0, T ; L^{2}(\Omega)\right)} \leqslant C_{4} .
$$

Combining this with (3.6), we observe by compactness that (3.12) is satisfied. This completes the proof of Theorem 3.4 .

\section{Acknowledgements}

My thanks are due to Professor Pierluigi Colli, Professor Nobuyuki Kenmochi, Professor Reiner Schätzle, and Professor Claudio Verdi for fruitful discussions. Moreover, I thank the referees for their valuable comments.

I would like to acknowledge the support of the Departments of Mathematics in the Universities of Pavia and Milan, where parts of this paper has been written. The financial support of the VIGONI programme 'Phase transitions, Stefan type problems, and minimizing movements' and the TMR project 'Viscosity solutions and their applications' is gratefully acknowledged.

\section{REFERENCES}

1. Alt, H. Lineare Funktionalanalysis. Springer, Berlin (1985).

2. AmAnn, H. Nonhomogeneous linear and quasilinear elliptic and parabolic boundary value problems. In: Schmeisser, H.-J. \& Triebel, H. (eds), Function Spaces, Differential Operators and Nonlinear Analysis. pp. 9-126. Teubner, Stuttgart (1993).

3. BARBU, V. Nonlinear Semigroups and Differential Equations in Banach Spaces. Noordhoff, Leyden (1976).

4. BRÉZIS, H. Monotonicity methods in Hilbert spaces and some applications to nonlinear partial differential equations. In: Zarantonello, E. (ed), Contributions to Nonlinear Functional Analysis. pp. 101-156. Academic, New York (1971).

5. Caffarelli, L., Crandall, M., Locan, M., \& Świech, A. On viscosity solutions of fully nonlinear equations with measurable ingredients. Comm. Pure Appl. Math. 49, (1996) 365-397. 
6. Caginalp, G. An analysis of a phase field model of a free boundary. Arch. Ration. Mech. Anal. 92, (1986) 205-245.

7. CaginalP, G. \& Lin, J. A numerical analysis of an anisotropic phase field model. IMA J. Appl. Math. 39, (1987) 51-66.

8. Colli, P., Frémond, M., \& Klein, O. Global existence of a solution to a phase field model for supercooling. Nonlinear Analysis, TMA, Series B, Real World Applications, 2, (2001) 523-539.

9. Colli, P. \& Laurençot, P. Weak solutions to the Penrose-Fife phase field model for a class of admissible heat flux laws. Phys. D 111, (1998) 311-334.

10. Colli, P., Laurençot, P., \& Sprekels, J. Global solution to the Penrose-Fife phase field model with special heat flux laws. Variations of Domain and Free-boundary Problems in Solid Mechanics (Paris, 1997). Kluwer, Dordrecht (1999) pp. 181-188.

11. Colli, P. \& Sprekels, J. J. Stefan problems and the Penrose-Fife phase field model. Adv. Math. Sci. Appl. 7, (1997) 911-934.

12. Colli, P. \& Sprekels, J. J. Weak solution to some Penrose-Fife phase-field systems with temperaturedependent memory. J. Diff. Equ. 142, (1998) 54-77.

13. Crandall, M., Ishit, H., \& Lions, P. Users's guide to viscosity solution of second order partial differential equations. Bull. Am. Math. Soc. 27, (1992) 1-67.

14. Damlamian, A. \& Kenmochi, N. Evolution equations associated with non-isothermal phase transitions. Functional Analysis and Global Analysis (Quezon City, 1996). Springer, Singapore (1997) pp. 62-77.

15. Damlamian, A. \& Kenmochi, N. Evolution equations generated by subdifferentials in the dual space of $\left(H^{1}(\Omega)\right)$. Discrete Contin. Dynam. Syst. 5, (1999) 269-278.

16. Elliott, C. M. \& Gardiner, A. Double obstacle phase field computations of dendritic growth, Research Report 96-19, University of Sussex, CMAIA, (1996).

17. Elliott, C. M., Gardiner, A., \& Kunn, T. Generalized double-obstacle phase field approximation of the anisotropic mean curvature flow, Research Report 96-17, University of Sussex, CMAIA, (1996).

18. Elliott, C. M., PAolini, M., \& SchÄtzle, R. Interface estimates for the fully anisotropic AllenCahn equation and anisotropic mean-curvature flow. Math. Models Methods Appl. Sci. 6, (1996) 1103 1118.

19. Elliott, C. M. \& Schätzle, R. The limit of the fully anisotropic double-obstacle Allen-Cahn equation in the nonsmooth case. SIAM J. Math. Anal. 28, (1997) 274-303.

20. Horn, W. A numerical scheme for the one-dimensional Penrose-Fife model for phase transitions. $A d v$ Math. Sci. Appl. 2, (1993) 457-483.

21. Horn, W., Laurençot, P., \& Sprekels, J. Global solutions to a Penrose-Fife phase-field model under flux boundary conditions for the inverse temperature. Math. Methods Appl. Sci. 19, (1996) 10531072.

22. Horn, W., Sprekels, J., \& Zheng, S. Global existence of smooth solutions to the Penrose-Fife model for Ising ferromagnets. Adv. Math. Sci. Appl. 6, (1996) 227-241.

23. Kenmochi, N. \& KUBO, M. Weak solutions of nonlinear systems for non-isothermal phase transitions. Adv. Math. Sci. Appl. 9, (1999) 499-521.

24. Kenmochi, N. \& NiezgódKA, M. Systems of nonlinear parabolic equations for phase change problems. Adv. Math. Sci. Appl. 3, (1993/94) 89-185.

25. KLEIN, O. Existence and approximation results for phase-field systems of Penrose-Fife type and Stefan problems, PhD thesis, Humboldt University, Berlin, (1997).

26. Klein, O. A semidiscrete scheme for a Penrose-Fife system and some Stefan problems in $\mathbb{R}^{3}$. Adv. Math. Sci. Appl. 7, (1997) 491-523.

27. Klein, O. A class of time-discrete schemes for a phase-field system of Penrose-Fife type. M2AN Math. Modelling Numer. Anal. 33, (1999) 1361-1292.

28. LaurençOt, P. Etude de quelques Problèmes aux Dérivées Partielles non Linéaires, PhD thesis, Université de Franche-Comté, (1993). 
29. Laurençot, P. Weak solutions to a Penrose-Fife model for phase transitions. Adv. Math. Sci. Appl. 5, (1995) 117-138.

30. Laurençot, P. Weak solutions to a Penrose-Fife model with Fourier law for the temperature. J. Math. Anal. Appl. 219, (1998) 331-343.

31. Marcus, M. \& Mizel, V. J. Complete characterization of functions which act, via superposition, on Sobolev spaces. Trans. Am. Math. Soc. 251, (1979) 187-218.

32. Penrose, O. \& Fife, P. Thermodynamically consistent models of phase-field type for the kinetics of phase transitions. Phys. D 43, (1990) 44-62.

33. SEKERKA, R. F. Role of instabilities in determination of the shapes of growing crystals. J. Cryst. Growth 128, (1993) 1-12.

34. Simon, J. Compact sets in the space $L^{p}(0, T ; B)$. Ann. Mat. Pura Appl. 4, (1987) 65-96.

35. SPREKELS, J. \& ZHENG, S. Global smooth solutions to a thermodynamically consistent model of phasefield type in higher space dimensions. J. Math. Anal. Appl. 176, (1993) 200-223.

36. TiBA, D. Optimal Control of Nonsmooth Distributed Parameter Systems. Springer, Berlin (1990).

37. WiLMANSKI, K. Private communication, (1999).

38. Zheng, S. Nonlinear parabolic equations and hyperbolic-parabolic coupled systems. Pitman Monographs and Surveys in Pure and Applied Mathematics, vol. 76. Longman, (1995). 